\title{
The polarization of $\mathrm{mm}$ methanol masers
}

\author{
H. Wiesemeyer ${ }^{1}$, C. Thum ${ }^{1}$, and C. M. Walmsley ${ }^{2}$ \\ ${ }^{1}$ Institut de Radio Astronomie Millimétrique, 300 rue de la Piscine, 38406 Saint Martin d'Hères, France \\ e-mail: wiesemey@iram.fr \\ 2 Osservatorio Astrofisico di Arcetri, Largo E. Fermi 5, 50125 Firenze, Italy
}

Received 26 February 2004 / Accepted 27 July 2004

\begin{abstract}
We present a survey of the polarization properties of mm-wavelength methanol masers, comprising both classes, and transitions from 84.5 to $157.0 \mathrm{GHz}$. Linear polarization is found in more than half of the observed objects, and circular

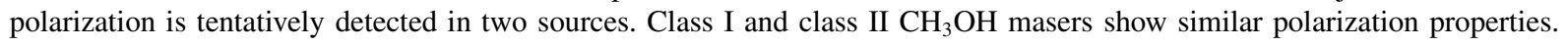
The largest linear polarization is found in the $133 \mathrm{GHz}$ class I maser towards L 379 (39.5\%), and in the $157 \mathrm{GHz}$ class II maser towards G 9.62+0.19 (36.7\%). The spectral profiles of the polarization angle of class I masers are mostly flat, except for two sources showing a linear slope. Since the mm-line methanol masers are expected to be weakly (or not) saturated, we suggest that the stronger fractional polarizations found by us are enhanced by anisotropic pumping and radiative losses. In NGC 7538, we find, for both maser classes, a good agreement between our polarization angles, and those measured for the submillimeter dust continuum. This can be taken as evidence for magnetic alignment of dust grains. It is also possible that an unsaturated maser with equally populated magnetic substates simply amplifies polarized continuum seed radiation. For class II masers, the polarization properties of the various velocity components towards a given source with detectable polarization are quite homogeneous. A possible explanation is discussed. Since methanol is non-paramagnetic, the circular polarization of the unsaturated maser emission can only be due to variations of the angle between the magnetic field and the line of sight along the maser propagation path.
\end{abstract}

Key words. masers - polarization - techniques: polarimetric - surveys - stars: formation - ISM: magnetic fields

\section{Introduction}

The polarization of astronomical masers is a complex field of current research. Whereas the polarization properties of non-amplified radiation simply derive from asymmetries of the emitting volume, masers introduce additional aspects due to their excitation and propagation. In terms of excitation, methanol masers can be classified into two well distinguished classes: class I sources (prototype Orion $\mathrm{BN} / \mathrm{KL}$ ) are loosely associated with, but clearly offset from ultracompact HII-regions. They may form in outflows and cloud-cloud encounters. These masers are thought to be pumped by collisions into high rotational levels from where they decay spontaneously into the upper level of the masing transition (Cragg et al. 1992). Class II sources (prototype W3 OH) are often coincident with known $\mathrm{OH}$ masers and HII-regions, and may thus occur in the compressed envelopes exterior to the ionized gas. Their masing transitions suggest pumping by FIR photons to torsionally excited levels (Sobolev \& Deguchi 1994).

Only two studies of methanol maser polarization properties are published (Caswell et al. 1993; Koo et al. 1988). Both surveys were made at $12 \mathrm{GHz}$ and cover only a small sample of class II masers. Linear polarization of up to $10 \%$ towards 4 (out of 11) and 2 (out of 5) sources respectively was detected in these two surveys.
Here we present a survey of polarized millimeter-wave methanol masers, including both maser classes. The polarization of class I masers was observed for the first time. The wealth of strong methanol masers detected at several $\mathrm{mm}$ transitions combined with the efficiency of a new polarimeter on the $30 \mathrm{~m}$ telescope were instrumental in improving upon the two $\mathrm{cm}$ surveys with respect to the number of detections of polarization, the better polarization sensitivity, and the advantage of doing all Stokes parameters simultaneously.

\section{Instrumentation and observations}

The observations were done at the IRAM $30 \mathrm{~m}$ telescope on Pico Veleta in February and May 2002. We used the observatory's $3 \mathrm{~mm}$ and $2 \mathrm{~mm}$ receivers (brought into phase with a common local oscillator reference) together with a versatile polarimeter working in the intermediate frequency band near $150 \mathrm{MHz}$. The inherent frequency agility was essential in measuring the polarization of several transitions quasi simultaneously. The polarimeter (described in more detail in Thum et al. 2003) derives all four Stokes parameters from the output signals of the two orthogonally polarized receivers, after insertion of phase lags of $0^{\circ}, 90^{\circ}, 180^{\circ}$ and $270^{\circ}$. For each of these phase lags, the coherent superposition of the two signals yields an 
Table 1. The observed methanol masers. All masers are in the torsional ground state. Rest frequencies are from Anderson et al. (1990).

\begin{tabular}{rrrrrlrrrrr}
\hline \hline$J_{\mathrm{u}}$ & $K_{\mathrm{u}}$ & & $J_{\mathrm{l}}$ & $K_{\mathrm{l}}$ & $\begin{array}{l}\text { Symm. } \\
\text { substate }\end{array}$ & $\begin{array}{r}\text { Frequency } \\
{[\mathrm{MHz}]}\end{array}$ & $\begin{array}{c}E_{\mathrm{u}} \\
{\left[\mathrm{cm}^{-1}\right]}\end{array}$ & $\begin{array}{c}E_{\mathrm{l}} \\
{\left[\mathrm{cm}^{-1}\right]}\end{array}$ & $\begin{array}{r}\text { Vel. resolution } \\
{\left[\mathrm{km} \mathrm{s}^{-1}\right]}\end{array}$ & $\begin{array}{c}\text { Maser } \\
\text { class }\end{array}$ \\
\hline 5 & -1 & $\rightarrow$ & 4 & 0 & $E$ & 84521.210 & 27.417 & 24.597 & 0.221 & $(I)$ \\
8 & 0 & $\rightarrow$ & 7 & 1 & $A^{+}$ & 95169.440 & 14.905 & 11.705 & 0.196 & $(I)$ \\
3 & 1 & $\rightarrow$ & 4 & 0 & $A^{+}$ & 107013.850 & 19.704 & 16.134 & 0.173 & $(I I)$ \\
6 & -1 & $\rightarrow$ & 5 & 0 & $E$ & 132890.790 & 37.092 & 32.660 & 0.140 & $(I)$ \\
6 & 0 & $\rightarrow$ & 6 & -1 & $E$ & 157048.620 & 42.331 & 37.092 & 0.119 & $(I I)$ \\
\hline
\end{tabular}

output signal whose power contains two terms, (i) the power from the two receivers; and (ii) a correlation term (like in an adding interferometer). The correlation terms are extracted by considering power differences between the four output channels, and yield Stokes $I, U$ and $V$. Stokes $Q$ is derived from the difference of the total powers of the two receivers. Small phase offsets between both receivers (of order $\$ 5^{\circ} / \mathrm{h}$ ) were regularly (typically once per hour) measured with a signal generator, and removed with a phase shifter acting on the local oscillator signal. The remaining instrumental polarization was regularly calibrated by means of unpolarized sources (mainly planets), for which all the output channels must yield the same signal strength. Any residual instrumental polarization on the optical axis was thus removed. The remaining polarization sidelobes mainly arise from the optics in the receiver cabin, and are well known (Thum et al. 2003). For the observations presented here, the beam polarization only limits the accuracy of the results for mispointed observations (see below), or masers spots scattered across a significant fraction of the polarization sidelobes.

Since the polarimeter's six output signals were analysed by the facility autocorrelator, we were restricted to use a $39 \mathrm{kHz}$ channel spacing, together with a $17.5 \mathrm{MHz}$ bandwidth. We used a parabolic apodization (Welch time lag window), yielding a half-power width of the spectral response function of $1.59 \times$ the channel separation, which is a good compromise between spectral resolution and sidelobe suppression. The resulting velocity resolutions are given in Table 1, together with the characteristics of the observed maser lines. The observed linewidth was approximately deconvolved from the spectral response to the parabolic apodization, using a Gaussian fit to the main lobe of the latter.

Our source selection is based on the strongest mm-wavelength masers found in the available surveys. Class I masers at mm wavelengths have been observed at 84.5 and $95 \mathrm{GHz}$ (Val'tts et al. 1995a) and $133 \mathrm{GHz}$ (Slysh et al. 1997), mm class II masers at $107 \mathrm{GHz}$ (Val'tts et al. 1995b) and $157 \mathrm{GHz}$ (Slysh et al. 1995). Many of the positions of class II masers are from the $6.6 \mathrm{GHz}$ survey of Menten (1991). Some mm methanol masers have been observed at the same velocities, but lower frequencies, with better positional accuracy (Kogan \& Slysh 1998, for $44 \mathrm{GHz}$ class I masers with the VLA; Minier et al. 2000, for 6.7 and $12.2 \mathrm{GHz}$ class II masers with the EVN and VLBA).
In order to avoid false polarization detections, we retain only those masers that are not affected by the instrumental polarization sidelobes, applying the following criteria:

(1) any mispointing results, even for an unpolarized signal, in an instrumental polarization. Since the latter is constant across the intermediate frequency band, a scaled copy of the Stokes $I$ signal would appear in Stokes $Q, U$ and $V$. In that case, the scaling factors must be compatible with the instrumental signature, known from our polarization sidelobe measurements. If this is the case, those data are discarded. For two or more masers offset within the beam, the result is not unique anymore, due to the convolution with the polarization sidelobes;

(2) wherever interferometric observations of $\mathrm{mm}$ - and other methanol masers indicate that the maser spots are scattered across the polarization sidelobes of the optical system of the $30 \mathrm{~m}$ telescope, we discard the data. This is the case e.g. for the two $84 \mathrm{GHz}$ maser components in DR21(OH) (Batrla \& Menten 1988).

The linear polarization given here is

$p_{\mathrm{L}}=\frac{\sqrt{Q_{\text {line }}^{2}+U_{\text {line }}^{2}}}{I_{\text {line }}}$

i.e. the linear polarization is quoted against the spectral line Stokes $I$ flux density (after subtraction of a contribution from the continuum emission). The relative error of the fractional linear polarization is (to first order in $p_{\mathrm{L}}$ ) given by the signal-to-noise ratio of the Stokes $I$ measurement. Continuum polarization measurements, as compared to spectral line polarimetry, are thus more precise, owing to the larger bandwidth. A few sources display continuum emission sufficiently strong to contribute to the signal in our quite narrow spectral band. The importance of dust polarization at $\mathrm{mm}$ wavelengths is still a matter of debate. Linear polarization (up to $8 \%$ ) of the dust continuum at $\lambda 1.3 \mathrm{~mm}$ was observed towards the Orion cloud core, offset from the BN/KL position (Leach et al. 1991). Towards BN/KL, the fractional polarization drops to $2.7 \%$ ("polarization hole"). In our case, a mispointing of 10 " can also lead to a linear polarization of up to $8 \%$ (depending on the direction of the mispointing). The fractional continuum polarization may also be affected by the measurement inaccuracy of Stokes $I$ due to fluctuations of the atmospheric emission (the 
Table 2. Detections ( $>3 \sigma_{\text {rms }}$ ) of linear polarization towards class $\mathrm{I} \mathrm{CH}_{3} \mathrm{OH}$ masers. Only velocity channels with the most significant polarization are given here (with velocities in Col. 10). For the other velocities, see Figs. 2 to 6. The maser polarization is given in Cols. 8 (fractional linear polarization $p_{\mathrm{L}}$ ) and 9 (polarization angle $\chi$, East from North). The maser fluxes and half-maximum full widths are derived from least-square fits of Gaussian components; the resulting linewidths are corrected for the instrumental broadening (for details see text).

\begin{tabular}{|c|c|c|c|c|c|c|c|c|c|c|}
\hline Source & Ref. & $\alpha_{(2000)}$ & & $\overline{\delta_{(2000)}}$ & $\begin{array}{r}\text { Frequency } \\
{[\mathrm{GHz}]}\end{array}$ & $\begin{array}{l}F_{v} \\
{[\mathrm{Jy}]}\end{array}$ & $\begin{array}{l}\Delta v_{F W H M} \\
{\left[\mathrm{~km} \mathrm{~s}^{-1}\right]}\end{array}$ & $\begin{array}{r}p_{\mathrm{L}} \\
{[\%]}\end{array}$ & $\begin{array}{r}\chi \\
{\left[{ }^{\circ}\right]}\end{array}$ & $\begin{array}{r}v_{\mathrm{lsr}} \\
{\left[\mathrm{km} \mathrm{s}^{-1}\right]}\end{array}$ \\
\hline OMC-2 & (1) & $05^{\mathrm{h}} 35^{\mathrm{n}}$ & ${ }^{\mathrm{n}} 27^{\mathrm{s}} .07$ & $-05^{\circ} 09^{\prime} 52^{\prime \prime} .5$ & 132.891 & 37.7 & 0.36 & $8.94 \pm 0.91$ & $22.1 \pm 2.6$ & 11.21 \\
\hline S 231 & (2) & 0539 & 13.06 & +354551.3 & 95.169 & 12.7 & 0.51 & $3.79 \pm 0.58$ & $118.1 \pm 4.5$ & -16.82 \\
\hline NGC 2264 & (1) & 0641 & 08.02 & +092940.0 & 132.891 & 14.0 & 0.62 & $10.6 \pm 1.7$ & $106.4 \pm 4.1$ & 7.22 \\
\hline M8E & (2) & 1804 & 53.76 & -242635.3 & 132.891 & 24.6 & 0.63 & $33.2 \pm 1.2$ & $154.56 \pm 0.8$ & 10.93 \\
\hline W 33-Met & (1) & 1814 & 11.08 & -175557.4 & 132.891 & 8.8 & 0.79 & $22.8 \pm 3.0$ & $154.5 \pm 3.4$ & 32.95 \\
\hline L 379 & (1) & 1829 & 24.69 & -151519.0 & 132.891 & 3.8 & 0.36 & $39.5 \pm 6.0$ & $133.7 \pm 3.4$ & 19.18 \\
\hline \multirow[t]{2}{*}{ W 51-Met 2} & (3) & 1923 & 46.50 & +142941.0 & 84.521 & 6.0 & 0.38 & $9.2 \pm 2.0$ & $23.6 \pm 5.6$ & 56.12 \\
\hline & & & & & 132.891 & 3.0 & 0.49 & $20.1 \pm 1.1$ & $10.2 \pm 3.4$ & 53.28 \\
\hline \multirow[t]{2}{*}{ DR 21-W } & (1) & 2038 & 54.72 & $+42 \quad 1922.4$ & 84.521 & 85.6 & 0.35 & $2.77 \pm 0.15$ & $48.1 \pm 1.4$ & -3.15 \\
\hline & & & & & 132.891 & 72.5 & 0.42 & $4.26 \pm 0.50$ & $7.7 \pm 3.0$ & -2.44 \\
\hline W 75 S(3) & (3) & 2039 & 03.50 & +422553.0 & 84.521 & 14.3 & 0.28 & $9.4 \pm 2.0$ & $7.8 \pm 5.7$ & -3.62 \\
\hline \multirow[t]{3}{*}{ NGC 7538-Met1 } & (3) & $23 \quad 13$ & 46.40 & +612733.0 & 84.521 & 3.8 & 0.70 & $5.5 \pm 1.6$ & $161.2 \pm 5.0$ & -58.00 \\
\hline & & & & & 95.169 & 14.1 & 0.82 & $14.5 \pm 1.3$ & $7.2 \pm 2.6$ & -57.42 \\
\hline & & & & & 132.891 & 3.5 & 0.81 & $24.6 \pm 1.8$ & $53.3 \pm 1.9$ & -57.35 \\
\hline
\end{tabular}

References: (1) VLA positions of the $44 \mathrm{GHz}$ maser, Kogan \& Slysh (1998); (2) VLBI positions of the 6.7 and $12.2 \mathrm{GHz}$ masers, Minier et al. (2000); (3) positions of the $95 \mathrm{GHz}$ survey of Val'tts (1995a).

IF polarimeter is not designed to measure weak continuum polarization levels). Stokes $Q, U$ and $V$ are not concerned, since they result from differential and correlation measurements, respectively, so our continuum polarization angle measurement remains reliable - on condition that the continuum emission is unresolved.

The statistical bias, introduced in the determination of $\sqrt{Q^{2}+U^{2}}$, was subtracted from the percentage polarization (see Wardle \& Kronberg 1974). The standard deviations of the linear polarization and its position angle were derived from the polarimeter's output signals by error propagation.

\section{Results}

In the following, the detections of methanol maser polarization are discussed within the context of the environment of their star-forming regions. The two maser classes are well separated, i.e. each of the transitions summarized in Table 1 shows maser action only in one of the two $\mathrm{CH}_{3} \mathrm{OH}$ maser classes. We find (all transitions confounded) polarization in 10 out of 14 class I sources satisfying our selection criteria, and in 3 out of 7 class II sources. We cannot break down this statistics into specific transitions, since not all sources were observed at all frequencies of Table 1. The distribution of polarization detections is shown in Fig. 1. The detections of linear polarization range between $2.8 \%$ and $39.5 \%$. The velocity profiles of polarization angles are either flat, or show a linear slope. Circular polarization is tentatively $\left(~ \gtrsim 3 \sigma_{\text {rms }}\right)$ detected towards two class I masers. Our results are summarized in Table 2 for class I mm $\mathrm{CH}_{3} \mathrm{OH}$ masers, respectively in Table 3 for their class II counterparts. Given the non-uniformity of our data set, and the fact that the accuracy of the polarization measurements depends on the signal-to-noise ratio, the upper limits for the non-detections depend much on the frequency and the weather conditions.

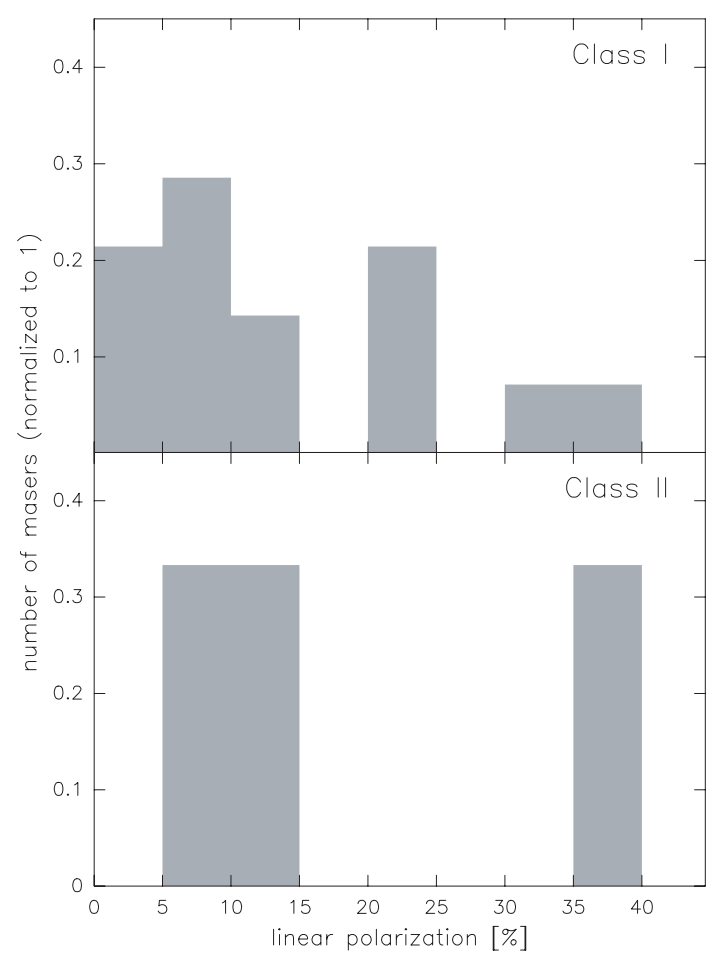

Fig. 1. The distribution of detections of linear polarization towards class I masers (top, for a total of ten sources) and class II masers (bottom, for three sources), binned into 5\% intervals.

Typical upper limits for non-detections range from $p_{\mathrm{L}}=0.5 \%$ to about $10 \%$.

\subsection{Class I masers}

The polarization spectra for class I masers are shown in Figs. 2 to 4, for $84 \mathrm{GHz}, 95 \mathrm{GHz}$, and $133 \mathrm{GHz}$, respectively. The 
Table 3. As Table 2, but for class $\mathrm{II} \mathrm{CH}_{3} \mathrm{OH}$ masers. The distinct main velocity components are listed separately.

\begin{tabular}{|c|c|c|c|c|c|c|c|c|c|}
\hline Source & Ref. & $\alpha_{(2000)}$ & $\delta_{(2000)}$ & $\begin{array}{r}\text { Frequency } \\
{[\mathrm{GHz}]}\end{array}$ & $\begin{array}{l}F_{v} \\
{[\mathrm{Jy}]}\end{array}$ & $\begin{array}{c}\Delta v \\
{\left[\mathrm{~km} \mathrm{~s}^{-1}\right]}\end{array}$ & $\begin{array}{r}p_{\mathrm{L}} \\
{[\%]}\end{array}$ & $\begin{array}{c}\chi \\
{\left[{ }^{\circ}\right]}\end{array}$ & $\begin{array}{r}v_{\mathrm{lsr}} \\
{\left[\mathrm{km} \mathrm{s}^{-1}\right]}\end{array}$ \\
\hline \multirow[t]{3}{*}{ NGC 7538-IRS1 } & (1) & $23^{\mathrm{h}} 13^{\mathrm{m}} 45^{\mathrm{s}} \cdot 36$ & $+61^{\circ} 28^{\prime} 09^{\prime} 7$ & 107.014 & $<16.3$ & $<2.34$ & $3.63 \pm 0.97$ & $104.5 \pm 6.8$ & -56.30 \\
\hline & & & & 107.014 & $<11.8$ & $<1.57$ & $5.7 \pm 1.2$ & $116.2 \pm 5.8$ & -58.59 \\
\hline & & & & 107.014 & 8.9 & 0.88 & $6.1 \pm 1.6$ & $112.6 \pm 6.7$ & -60.56 \\
\hline \multirow[t]{4}{*}{ Cep A } & (1) & $2256 \quad 18.10$ & +620149.5 & 157.049 & 19.5 & 0.48 & $12.3 \pm 1.7$ & $86.4 \pm 4.0$ & -3.84 \\
\hline & & & & 157.049 & 40.6 & 0.34 & $14.10 \pm 0.94$ & $87.2 \pm 2.0$ & -2.80 \\
\hline & & & & 157.049 & 17.0 & 0.57 & $14.0 \pm 2.0$ & $79.0 \pm 4.2$ & -1.75 \\
\hline & & & & 157.049 & 10.2 & 0.58 & $10.6 \pm 2.7$ & $81.2 \pm 6.9$ & -1.30 \\
\hline \multirow[t]{2}{*}{ G $9.62+0.19$} & (1) & $\begin{array}{lll}18 & 06 & 14.80\end{array}$ & -203132.0 & 157.049 & 6.4 & 0.88 & $31.5 \pm 3.7$ & $142.9 \pm 3.1$ & 1.55 \\
\hline & & & & 157.049 & 3.5 & 0.66 & $36.7 \pm 5.7$ & $135.1 \pm 4.8$ & -0.09 \\
\hline
\end{tabular}

Note: The masers of NGC 7538-IRS1 are blended with thermal emission, only upper limits can be given for flux and linewidth. References: (1) VLBI positions of Minier et al. (2000).

spectra of the fractional linear polarization (in percent) and its position angle are for the velocity range indicated by the greyshadowed area in the Stokes $I$ spectra. All polarization angles are given in counterclockwise sense ( $\mathrm{E}$ from $\mathrm{N})$.

\subsubsection{OMC-2}

We observed this maser, located in a cluster of infrared sources and far-infrared condensations with multiple outflow activity, in the $133 \mathrm{GHz} 6_{1} \rightarrow 5_{0}$ transition of E-type methanol (first detection by Slysh et al. 1997). Other class I transitions were previously observed, but with unreliable astrometry. The maser peaks at $11.35 \mathrm{~km} \mathrm{~s}^{-1}$, the strongest polarization $\left(p_{\mathrm{L}}=8.9 \%\right)$ is observed at $11.21 \mathrm{~km} \mathrm{~s}^{-1}$. At first sight, the profile of the linear fractional polarization looks like a scaled copy of the Stokes $I$ line profile. However, the polarized feature is significantly narrower than the maser component $\left(0.37 \mathrm{~km} \mathrm{~s}^{-1} \mathrm{vs}\right.$. $0.69 \mathrm{~km} \mathrm{~s}^{-1}$, respectively). An instrumental origin can also be ruled out, since criterion (1) of Sect. 2 does not apply. The polarization angle $\left(22^{\circ}\right)$ remains constant across the line profile. As a matter of fact, the outflow driven by the source FIR 3 and mapped by Williams et al. (2003) has a position angle of $30^{\circ}$; the brightest maser spots of the $7_{0} \rightarrow 6_{1} A^{+} \mathrm{CH}_{3} \mathrm{OH}$ maser (at $44 \mathrm{GHz}$, mapped with the VLA by Kogan \& Slysh 1998) are on a straight line of position angle $32^{\circ}$ (E from N). Both position angles are close to our polarization angle. This interesting finding, schematically shown in Fig. 7, will be discussed below. The position and velocity of the maser spike corresponds to one of the components of the $44 \mathrm{GHz}$ maser (component number 7 of Kogan \& Slysh). The thermal linewings make it impossible to know whether we also get weaker maser emission at the velocities of the other VLA maser spots, since the velocity dispersion of the latter is merely $0.23 \mathrm{~km} \mathrm{~s}^{-1}$. If so, they could produce an important instrumental polarization, because they are spread over a N-S line extending over $15^{\prime \prime}$, i.e. as large as the telescope's main beam at that frequency. However, this would not be compatible with our flat polarization angle profile, since the sidelobe polarization pattern is significantly different in Stokes $Q$ and $U$ (see Thum et al. 2003).

\subsubsection{S 231}

The $95 \mathrm{GHz} 8_{0} \rightarrow 7_{1} A^{+} \mathrm{CH}_{3} \mathrm{OH}$ emission, first observed by Val'tts (1995a), is at $-16.8 \mathrm{~km} \mathrm{~s}^{-1}$, with a $3.8 \%$ linear polarization. Its polarization angle across the main component shows a clearly defined gradient, a finding that will be discussed in the next section. A second, weaker maser component, not or weakly polarized, is at $-15.2 \mathrm{~km} \mathrm{~s}^{-1}$, at the blue-shifted end of the cluster of $6.7 \mathrm{GHz} 5_{1} \rightarrow 6_{0} A^{+} \mathrm{CH}_{3} \mathrm{OH}$ masers (EVN observations of Minier et al. 2000).

\subsubsection{NGC 2264}

The $133 \mathrm{GHz} 6_{-1}-5_{0} E \mathrm{CH}_{3} \mathrm{OH}$ maser, first observed by Slysh et al. (1997), is located in a core of this massive star-forming region, that is associated with the bright infrared source IRS 1 (Allen 1972). The position angle of the linear polarization $\left(\sim 110^{\circ}\right)$ remains remarkably constant. Schreyer et al. (1997) decomposed the outflow activity of IRS 1 into two flows. They infer that one of them, extending in North-South direction, is driven by IRS 1 and seen nearly pole-on. The $133 \mathrm{GHz}$ $\mathrm{CH}_{3} \mathrm{OH}$ maser is located in that outflow.

\subsubsection{M8E}

We find in the $133 \mathrm{GHz}$ line linear polarization as high as $33 \%$. Its position angle of $155^{\circ}$ is flat across the spectral profile. M8E harbours a strong $44 \mathrm{GHz}$ maser (Kogan \& Slysh (1998), whose two components are 1."7 apart (2500 AU at a distance of $1.5 \mathrm{kpc}$, see Val'tts 1999a). The $133 \mathrm{GHz}$ maser is at the radial velocity of the strongest $44 \mathrm{GHz}$ component $\left(v_{\mathrm{lsr}}=10.94 \mathrm{~km} \mathrm{~s}^{-1}\right.$, the weaker one at $v_{\mathrm{lsr}}=10.45 \mathrm{~km} \mathrm{~s}^{-1}$ does not appear at $133 \mathrm{GHz}$ ). At $10.84 \mathrm{~km} \mathrm{~s}^{-1}$, we find a circular polarization of $p_{\mathrm{C}}=(-7.1 \pm 1.0) \%$, which cannot be due to the sidelobe polarization alone (Fig. 10). The maser is most 

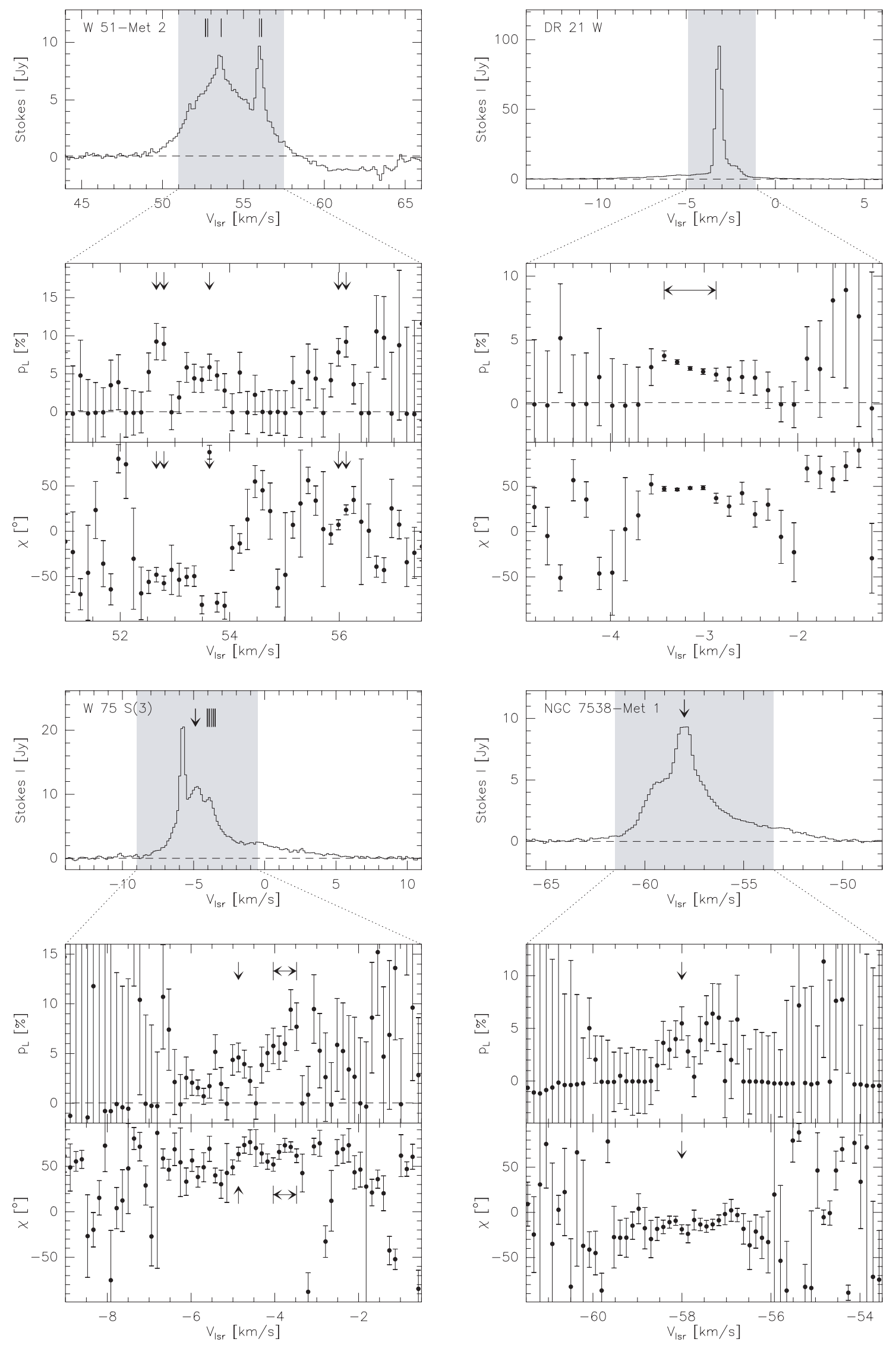

Fig. 2. Class I methanol masers at $84 \mathrm{GHz}$. For each source, Stokes $I$ (top), the fractional linear polarization $p_{\mathrm{L}}$ (center), and the polarization angle $\chi(\mathrm{E}$ from $\mathrm{N}$, bottom) are shown, with spectral baselines subtracted. Vertical bars or arrows and horizontal arrows mark the velocities or velocity ranges, respectively, with $p_{\mathrm{L}}>3 \sigma_{\text {rms }}$. The grey-shaded areas in the Stokes $I$ spectra indicate the velocity range for $p_{\mathrm{L}}$ and $\chi$. 

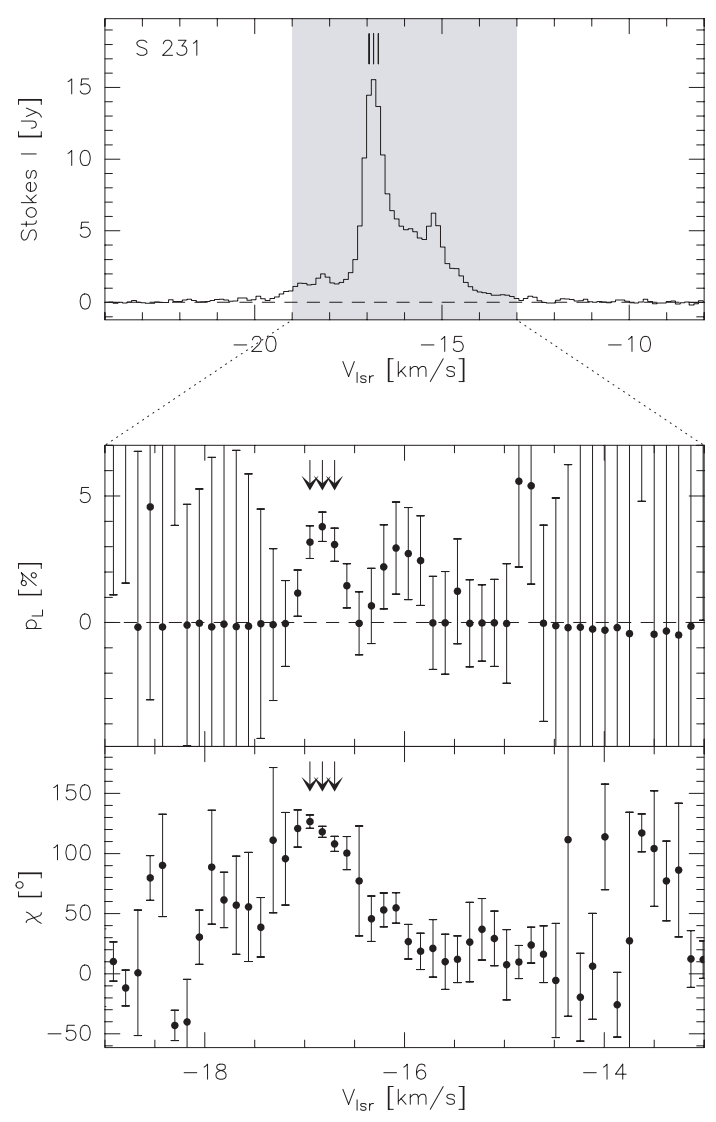

Fig. 3. Class I methanol masers at $95 \mathrm{GHz}$.

likely not associated with the $\mathrm{CO}$ outflow (position angle $54^{\circ}$, W from N, Mitchell et al. 1992), since the velocities of the quiescent gas and the methanol maser are close to each other (since the outflow almost appears pole-on, a large velocity offset would be expected for an associated maser). Consequently, Val'tts (1999a) suggests that the class I maser is located in the front of the bipolar outflow at an interface with the surrounding dense molecular gas.

\subsubsection{W 33-Met}

We measure, for this $133 \mathrm{GHz}$ maser, a fractional linear polarization of $23 \%$ at $32.9 \mathrm{~km} \mathrm{~s}^{-1}$. The radial velocity coincides with that of the southern component of the two VLA $44 \mathrm{GHz}$ maser spots of Kogan \& Slysh (1998). Another polarized feature, with $p_{\mathrm{L}} \sim 10 \%$, is at a radial velocity of about $37 \mathrm{~km} \mathrm{~s}^{-1}$, where the $44 \mathrm{GHz}$ maser has a second component, at about $32^{\prime \prime}$ northwards. This feature coincides with a broader $\mathrm{CH}_{3} \mathrm{OH}$ emission, of equal flux density. Val'tts (1999b) suggests that this (spatially extended) component is a quasimaser with a low gain and weak line narrowing. Thus, the linear polarization measured by us (although barely significant, $\sim 3 \sigma_{\text {rms }}$ ) towards this component almost certainly has an important instrumental contribution.

No spatially resolved map of the outflow exists. Val'tts (1999b) find the class I maser to be associated with a CS condensation, containing a very young protostar.
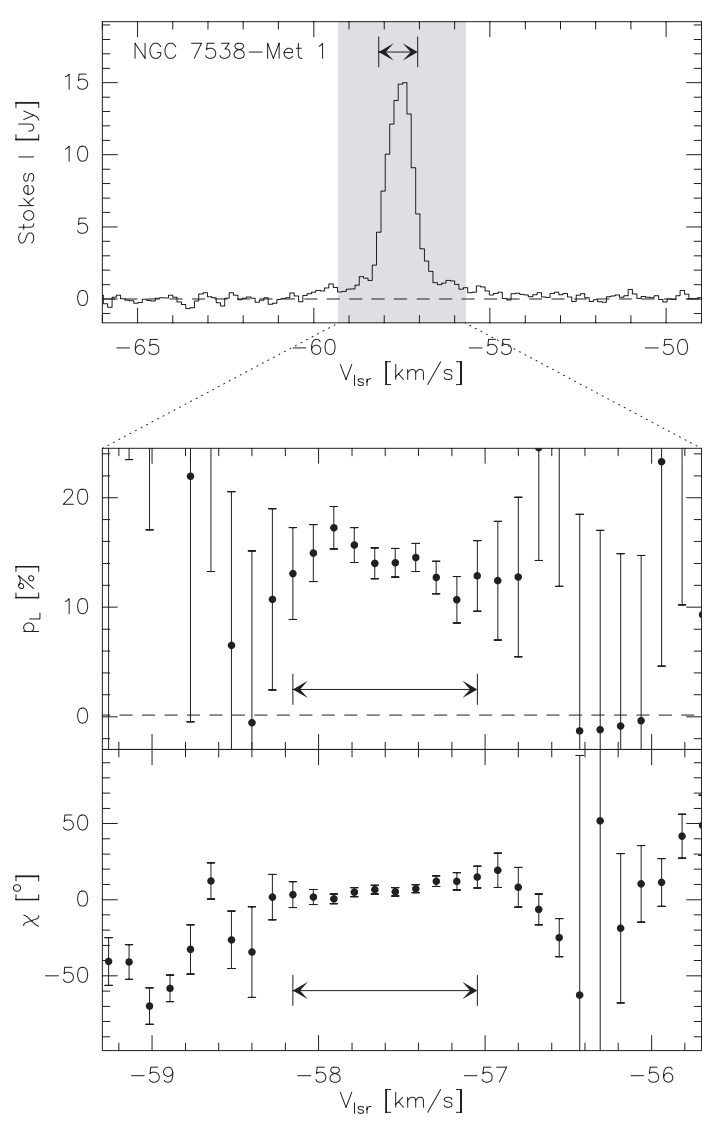

\subsubsection{379}

The $133 \mathrm{GHz}$ maser is located $30^{\prime \prime}$ to the north of IRAS source L 379-IRS1. From the absence of linear polarization in the thermal red-shifted linewing, it is excluded that the measured linear polarization of $39 \%$ at a radial velocity of $19.2 \mathrm{~km} \mathrm{~s}^{-1}$ is due to the telescope's sidelobe polarization. The polarization angle has a flat spectral profile with $\chi=134^{\circ}$. This $133 \mathrm{GHz}$ maser concides with the northern $\lambda 800 \mu \mathrm{m}$ continuum peak, discovered by Kelly \& MacDonald (1996), and is probably associated with their $\mathrm{CO}$ outflow, whose direction is at $75^{\circ}$. It is likely that the red-shifted linewing consists of a blend of (quasi-) maser emission. As a matter of fact, we find an even stronger fractional linear polarization with a similar polarization angle at about $19.6 \mathrm{~km} \mathrm{~s}^{-1}$, but of lower sig-

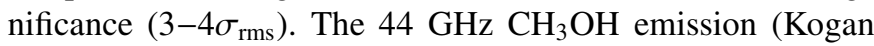
\& Slysh 1998) shows a total of 11 masers, scattered across a region of $46^{\prime \prime}$ in diameter, in two groups at radial velocities of $18.8-20.8 \mathrm{~km} \mathrm{~s}^{-1}$ and $16.3-18.8 \mathrm{~km} \mathrm{~s}^{-1}$, respectively. From the latter group, we detect $\mathrm{CH}_{3} \mathrm{OH}$ emission, whose line profile can be decomposed into two Gaussian spectral components, a large one (linewidth $7.0 \mathrm{~km} \mathrm{~s}^{-1}$ FWHM), and a narrower one, with a linewidth of $2.1 \mathrm{~km} \mathrm{~s}^{-1}$ (at best a quasi-maser without substantial line narrowing). The polarization of these components, most likely scattered across our beam, is strong across the whole line profile. Stokes $U$ is an inverted and scaled copy of Stokes $I$, and the signs of the other Stokes parameters agree with the instrumental signature. We thus discard this polarization signal as of instrumental origin. 

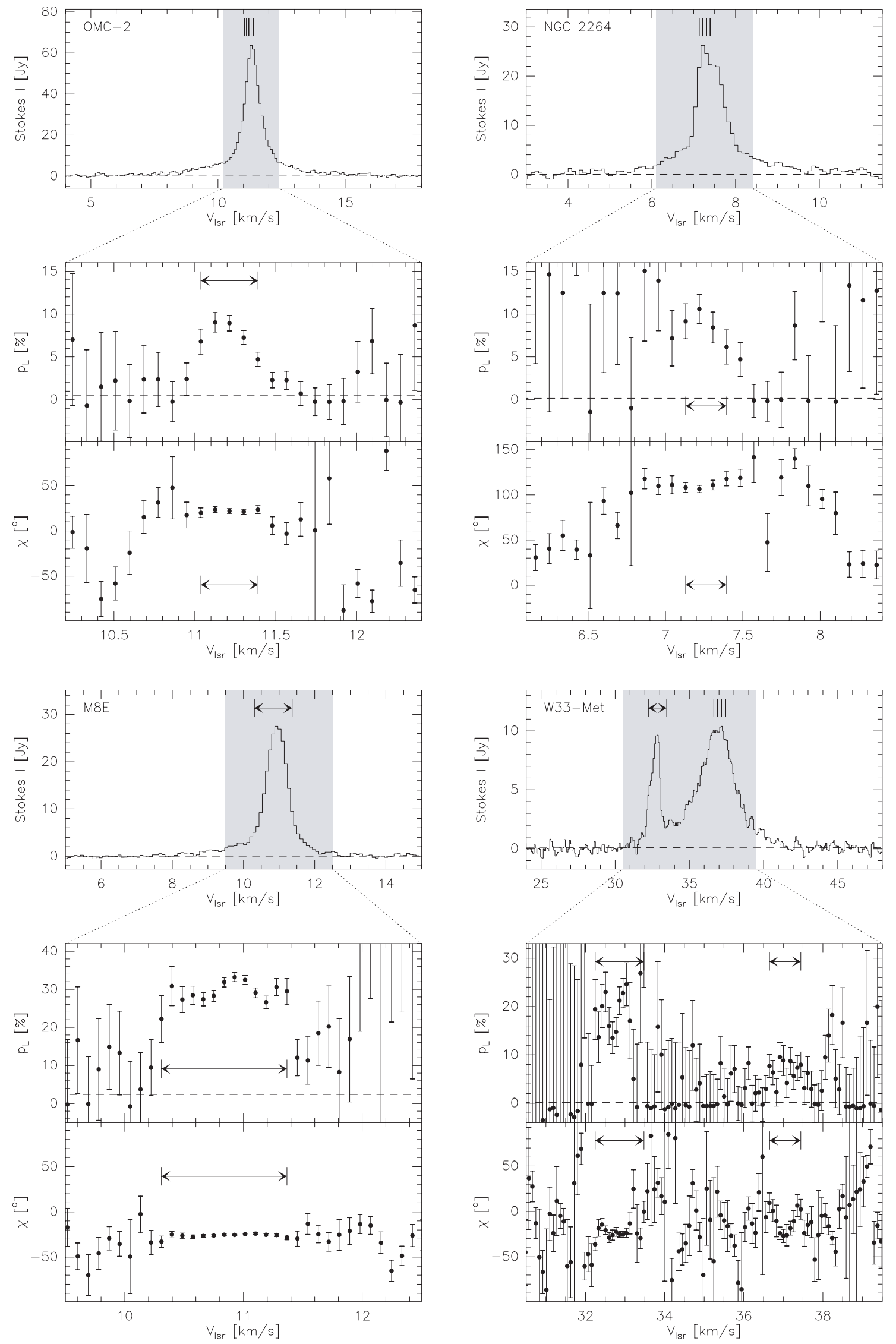

Fig. 4. Class I methanol masers at $132.9 \mathrm{GHz}$. 

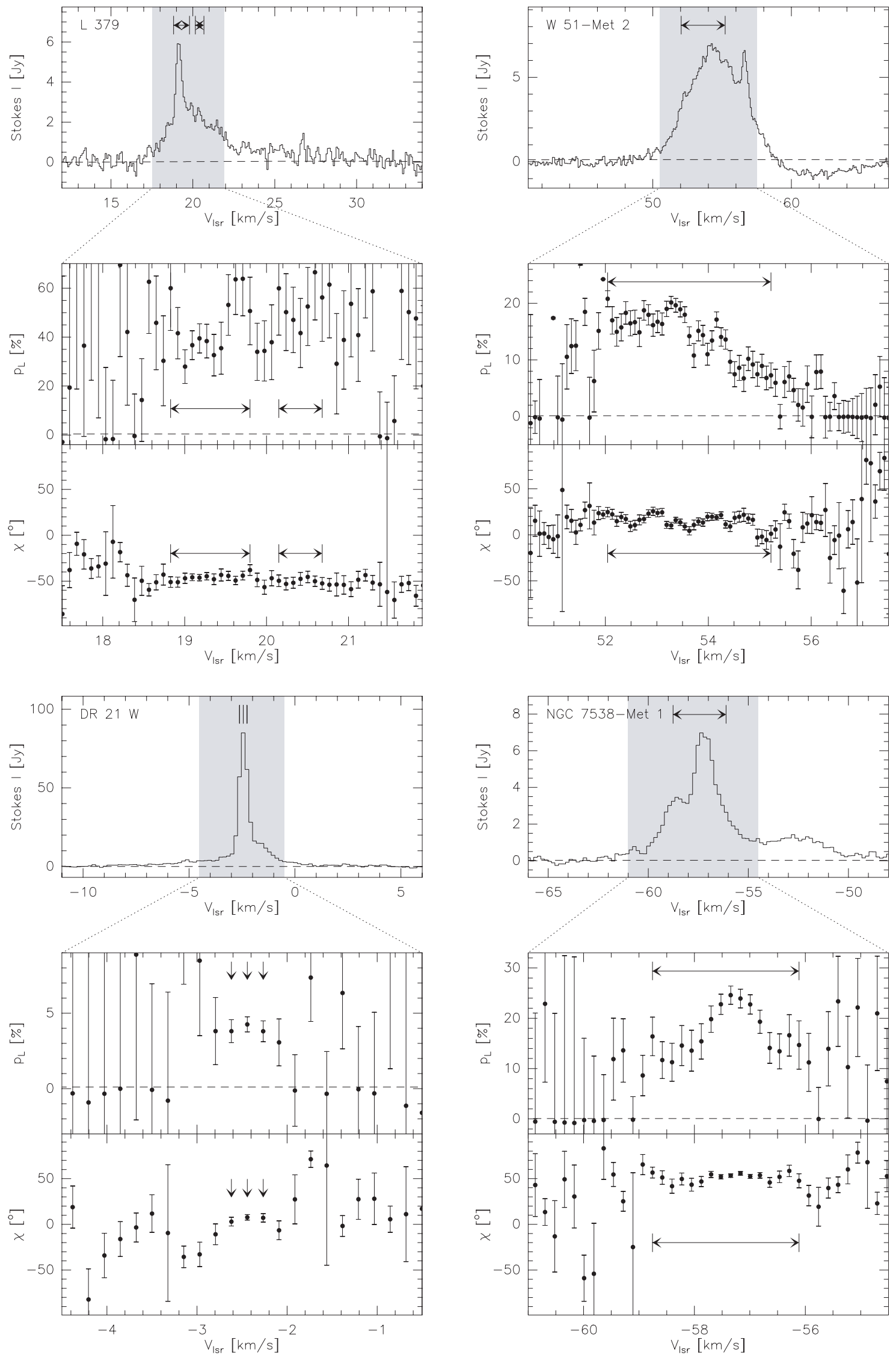

Fig. 4. continued. 


\subsubsection{W51-Met2}

The $84 \mathrm{GHz}$ line of this $\mathrm{CH}_{3} \mathrm{OH}$ maser is blended with a broad thermal emission. However, the linear polarization disappears across a large part of the thermal profile, except for the single-peaked maser line at $56.1 \mathrm{~km} \mathrm{~s}^{-1}$, and another component at $53.3 \mathrm{~km} \mathrm{~s}^{-1}$ (possible maser emission masked by the thermal emission, cf. Fig. 2). The spectral profile of the polarization angle of the $84 \mathrm{GHz}$ maser spike has a linear slope, as in the case of $\mathrm{S} 231$. Jumps in the polarization angle of $90^{\circ}$ at certain velocities are due to a statistical bias in the weak-signal limit. The thermal emission of the $133 \mathrm{GHz}$ maser is strongly polarized (between 52.0 and $55.2 \mathrm{~km} \mathrm{~s}^{-1}$, reaching a maximum of $20.1 \%$ at $53.3 \mathrm{~km} \mathrm{~s}^{-1}$, with a polarization angle of $-5^{\circ}$ to $30^{\circ}$ in this velocity range). The line peak can be decomposed into a broad component (linewidth $4.5 \mathrm{~km} \mathrm{~s}^{-1}$ FWHM) and a narrow one (linewidth $0.482 \mathrm{~km} \mathrm{~s}^{-1}$ ). We consider it quite possible that this extraordinary, broad polarization profile may be due to an anisotropic optical depth (Goldreich \& Kylafis 1982). The amplification of polarized background continuum serving as "seed" is also possible, given the flux density ratio of $F_{\text {line }} / F_{\text {cont }}=11$ at $53.3 \mathrm{~km} \mathrm{~s}^{-1}$ (or 2.8 if we only consider the narrow component). However, the uncertain astrometry and extent of the thermal emission could introduce an important instrumental contribution, too (as discussed in the previous section), which makes impossible an unbiased detection of the polarization of the $133 \mathrm{GHz}$ maser at $56.6 \mathrm{~km} \mathrm{~s}^{-1}$ (the linear polarization is not significant, as opposed to the $84 \mathrm{GHz}$ maser).

As an indication of the possibility of polarized seed radiation, we detect a fractional linear continuum polarization of $p_{\mathrm{L}}=(4.7 \pm 1.2) \%$ at $84 \mathrm{GHz}$, and $p_{\mathrm{L}}=(13.7 \pm 2.1) \%$ at $133 \mathrm{GHz}$ (with $\chi=18.4$, not too far from the submm measurement, Chrysostomou et al. 2002), respectively. Note that the errors in $p_{\mathrm{L}}$ do not contain the uncertainty due to atmospheric fluctuations (as discussed in Sect. 2).

The host source is the W51 f subregion of the W $51 \mathrm{~A}$ star forming cloud (see Sievers et al. 1991, for a $\lambda 870 \mu \mathrm{m}$ and $\lambda 1.3 \mathrm{~mm}$ map). Submillimeter continuum polarimetry (Chrysostomou et al. 2002) reveals a marginal linear dust polarization of $2.5 \%$, at a position angle $24^{\circ}$. The authors conclude that the overall magnetic field is rather weak, and becomes only important close to the sites of star formation. The lack of evidence for a continuum signal at longer wavelengths made it difficult to reveal the nature of this maser's environment. Hodapp \& Davis (2002) recently found a spatially unresolved, but strong $\mathrm{H}_{2}(1-0) \mathrm{S}(1)$ line $(\lambda 2.12 \mu \mathrm{m})$ about $10^{\prime \prime}$ eastwards of the methanol maser position. Taking into account the uncertain astrometry (no interferometer observations are available), this emission could come from the same shocked gas that harbours the methanol maser, in the immediate vicinity of a star enshrouded by the molecular and dust cloud.

\subsubsection{DR21-W}

DR 21-W is one of three sources in which we heave measured and detected linear polarization in both the $84 \mathrm{GHz}$ and $133 \mathrm{GHz}$ line. They both have flat polarization angle profiles (with position angles $48^{\circ} .1$ and 7.7 respectively). They are single-peaked, and are rather weakly polarized (about 3-4\%). At $133 \mathrm{GHz}$, we find a significant circular polarization of $p_{\mathrm{C}}=(3.52 \pm 0.68) \%$ at $v_{\mathrm{lsr}}=-2.63 \mathrm{~km} \mathrm{~s}^{-1}$ (Fig. 10), slightly offset from the velocity of maximal linear polarization $\left(-2.44 \mathrm{~km} \mathrm{~s}^{-1}\right)$. As in the case of M8E, this detection can not be entirely attributed to the sidelobe polarization.

Kogan \& Slysh (1998) observed the $7_{0}-6_{1} A^{+}$methanol maser at $44 \mathrm{GHz}$ with the VLA, and find two major components separated by 2 '. 8 , whose radial velocities are close to ours. The environment of this maser is fairly well known: Davis \& Smith (1996) obtained high-resolution images in $\mathrm{H}_{2}$ (2.12 $\mu$ m emission). Their spectrum closest to the methanol maser is best described by a C-type planar shock. The submillimetre continuum polarimetry (Minchin \& Murray 1994) reveals a linear magnetic field, in position angle $\left(114^{\circ}\right)$ intermediate between the cloud core and the dominant outflow axis $\left(70^{\circ}\right.$, Garden et al. 1991). This polarization angle, however, does not agree well with that of either of the two maser lines. Liechti \& Walmsley (1997) observed the thermal $2_{\mathrm{k}} \rightarrow 1_{\mathrm{k}}$ emission of $A^{+}$and $E$-type methanol. The methanol maser shown here coincides in position with a thermal clump in $2_{1} \rightarrow$ $1_{1} E$ emission, whose major axis is at position angle $45^{\circ}-$ remarkably close to our $84 \mathrm{GHz}$ polarization angle. The thermal methanol emission also appears in our $84 \mathrm{GHz}$ and $133 \mathrm{GHz}$ spectra as a broad, unpolarized pedestal underneath the maser lines.

\subsubsection{W75 S(3)}

In this source, located in the same ridge of molecular gas as the DR 21 region (Wilson \& Mauersberger 1990), the $84 \mathrm{GHz}$ masers consist of a narrow main component, and a blend of several, weaker (quasi-) maser components, together with a broad underlying thermal emission. Only the polarization of one of the weaker components is significant, the other maxima of the polarization profile are far less significant (see Fig. 2). It is difficult to distinguish this feature from an intrinsic or instrumental polarization of the underlying, broad emission. The profile of the polarization angle oscillates between $50^{\circ}$ and $80^{\circ}$. At $133 \mathrm{GHz}$, no significant linear polarization was detected.

\subsubsection{NGC 7538-Met1}

This is the only source in our sample with a bona-fide detection of maser polarization in each of the three class I maser frequencies. The polarization angles at the maser peaks are different, their velocities are within $0.8 \mathrm{~km} \mathrm{~s}^{-1}$. This difference is most likely intrinsic, since the polarization angle profiles themselves have a peak-to-peak variation of up to $20^{\circ}$. The strongest polarization is found at $133 \mathrm{GHz}$ (24.6\%), resulting in the most significant polarization angle measurement among the three transitions. The polarization angle has a remarkably flat spectral profile (position angle $53^{\circ} .3$ at the $133 \mathrm{GHz}$ maser spike, as compared to $161^{\circ} 2$ at $84 \mathrm{GHz}$, and 7.2 at $95 \mathrm{GHz}$ ). Although part of the polarization could come from the underlying broad emission (at $84 \mathrm{GHz}$ and $133 \mathrm{GHz}$ ), it is unlikely that the latter reaches a fractional polarization as high as $25 \%$. The 
$\lambda 870 \mu \mathrm{m}$ continuum polarization is from magnetically aligned dust grains (Momose et al. 2001). Its position angle (41:8) differs by 11.5 from our measurement at $133 \mathrm{GHz}$ (Fig. 8).

\subsection{Class II masers}

The polarization spectra for class II masers are shown in Figs. 5 and 6, for $107 \mathrm{GHz}$ and $157 \mathrm{GHz}$, respectively.

\subsubsection{NGC 7538-IRS1}

The $107 \mathrm{GHz}$ maser from this source is located $37^{\prime \prime}$ northwards of its class I counterpart, and coincides with the infrared source IRS 1 . The $107 \mathrm{GHz}$ methanol maser has three components, each of similar polarization. The $107 \mathrm{GHz}$ continuum of the source, as seen in our spectra, is not polarized (upper limit $p_{\mathrm{L}}<0.4 \%$ ). The velocities of the maser spikes correspond to those of the $6.7 \mathrm{GHz}$ maser components $\mathrm{A}, \mathrm{E}$, and D (or C) of Minier et al. (2000), which are 0.' 6 apart (i.e. $1620 \mathrm{AU}$ at $2.7 \mathrm{kpc}$ distance). The polarization angle of the three velocity components of the $107 \mathrm{GHz}$ maser (Fig. 5) is $105^{\circ}-116^{\circ}$, not too far from the position angle of the aligned $6.7 \mathrm{GHz}$ masers of subgroup $\mathrm{A}$, and close to the position angle of the equator of the edge-on circumstellar disk proposed by Minier et al. They suggest that the maser resides at a single radius of this disk. This conjecture is corroborated by the detection of an elongated structure seen in an interferometric ${ }^{13} \mathrm{CO}(1-0)$ map (Scoville et al. 1986), perpendicular to the bipolar H II region, and at an angle of $55^{\circ}$ to the major outflow axis. As the class I maser to the south of IRS 1, our class II maser polarization angle agrees more or less (within $10^{\circ}$ to $20^{\circ}$ ) with that of the $\lambda 870 \mu \mathrm{m}$ dust emission (Momose et al. 2001, see our Fig. 8). The linear polarization at $107 \mathrm{GHz}$ yields significant results for three velocity components ( $p_{\mathrm{L}}=3.6$ to $6.1 \%$, Fig. 5). However, given that this fractional polarization is rather weak (for the $157 \mathrm{GHZ}$ maser, no significant polarization is detected), it cannot be excluded that the whole line profile, with both (quasi-) maser and thermal emission is polarized, due to the amplification of a polarized continuum source. As a matter of fact, the continuum emission at $157 \mathrm{GHz}$ has a linear polarization of $p_{\mathrm{L}}=(8.4 \pm 1.0) \%$. Its position angle $(-84.0 \pm 2.7)$ is close to that of the $\lambda 870 \mu \mathrm{m}$ polarization ( $-87^{\circ} .2$, Momose et al.), which differs from the polarization angle of the $107 \mathrm{GHz}$ maser by about $23^{\circ}$. For the ratio of the line to continuum brightness temperature we estimate values of 6 for the $107 \mathrm{GHz}$ maser, and of 5 for the $157 \mathrm{GHz}$ maser, respectively.

\subsubsection{Cep A}

We observed the $107 \mathrm{GHz}$ and $157 \mathrm{GHz}$ masers towards Cep A East, detecting a significant linear polarization only from the latter. This source contains masers at four well separated velocities, which agree with the components of the $12.2 \mathrm{GHz}$ maser (VLBI observation of Minier et al. 2000). The fractional polarization of the components of the $157 \mathrm{GHz}$ masers ranges from $p_{\mathrm{L}}=10.6 \%$ to $14.1 \%$, and the polarization angle from $79^{\circ}$ to $86^{\circ}$ (see Fig. 11). The significant polarization
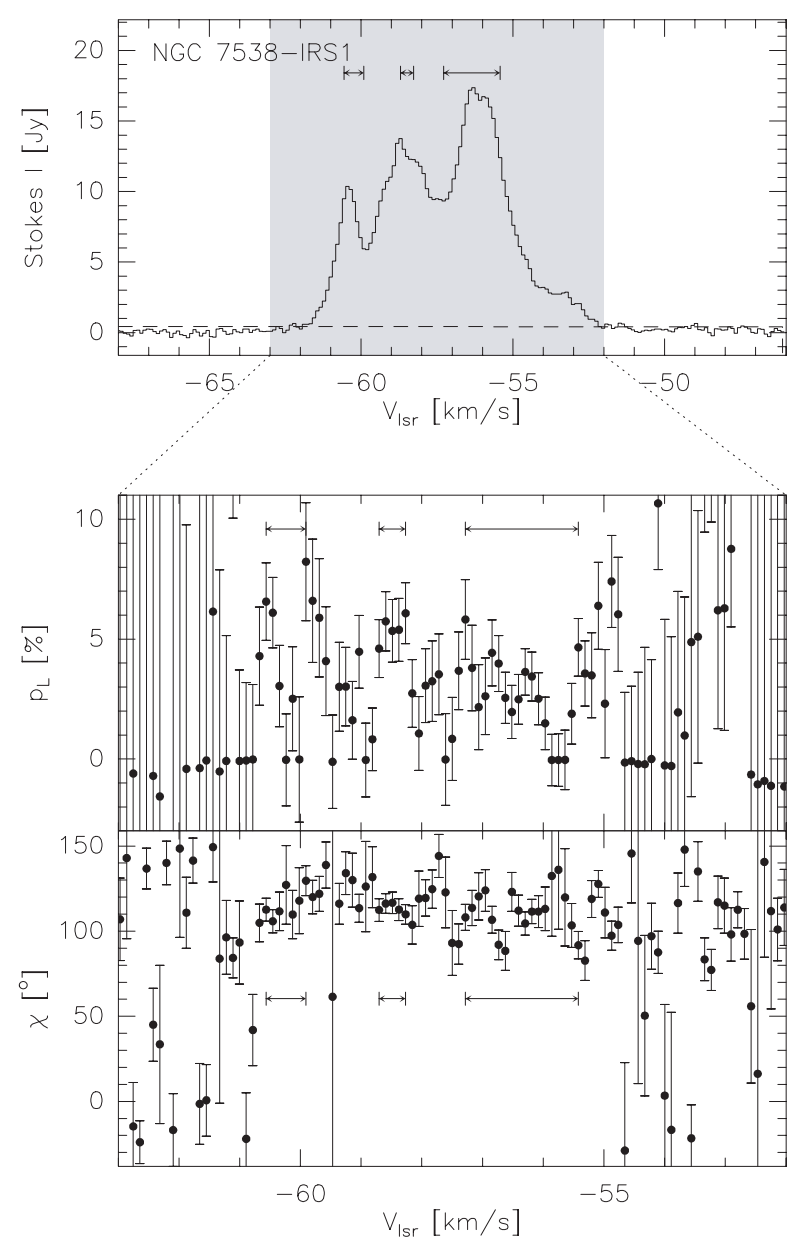

Fig. 5. The $107 \mathrm{GHz}$ polarization spectra of NGC 7538-IRS1, a class II $\mathrm{CH}_{3} \mathrm{OH}$ maser.

measurements (i.e. $p_{\mathrm{L}}>3 \sigma$ ) are clearly correlated with the maser spikes (there is stronger, but less significant polarization between the spikes). The velocity of the first component listed in Table 3 agrees well with that of component B in the interferometer map of the $107 \mathrm{GHz}$ maser (Mehringer et al. 1997). Our last two components correspond in both velocity and flux density to component A of Mehringer et al. (after smoothing to their spectral charactersistics), which is spectrally unresolved by them, but asymmetric due to the blend of the two spikes. Although Mehringer et al. infer from their results that the $107 \mathrm{GHz}$ masers arise from regions with slightly different physical characteristics, their polarization properties are rather homogeneous - although the projected distance between components A and B is 0.'7 (corresponding to $500 \mathrm{AU}$ at a distance of $730 \mathrm{pc}$, Torrelles et al. 1996). The fourth component at $v_{\text {lsr }}=-2.8 \mathrm{~km} \mathrm{~s}^{-1}$ does not have a counterpart at $107 \mathrm{GHz}$. At $157 \mathrm{GHz}$, it is the strongest one (both in intensity and polarization). The velocities of all these masers are redshifted with respect to those of the ambient molecular cloud and the expanding bubble of $\mathrm{H}_{2} \mathrm{O}$ masers (Torrelles et al. 2001). According to Minier et al. (2000), the methanol masers and the water maser are located in the same environment. However, given the complexity of the region, it cannot be ruled out that the 

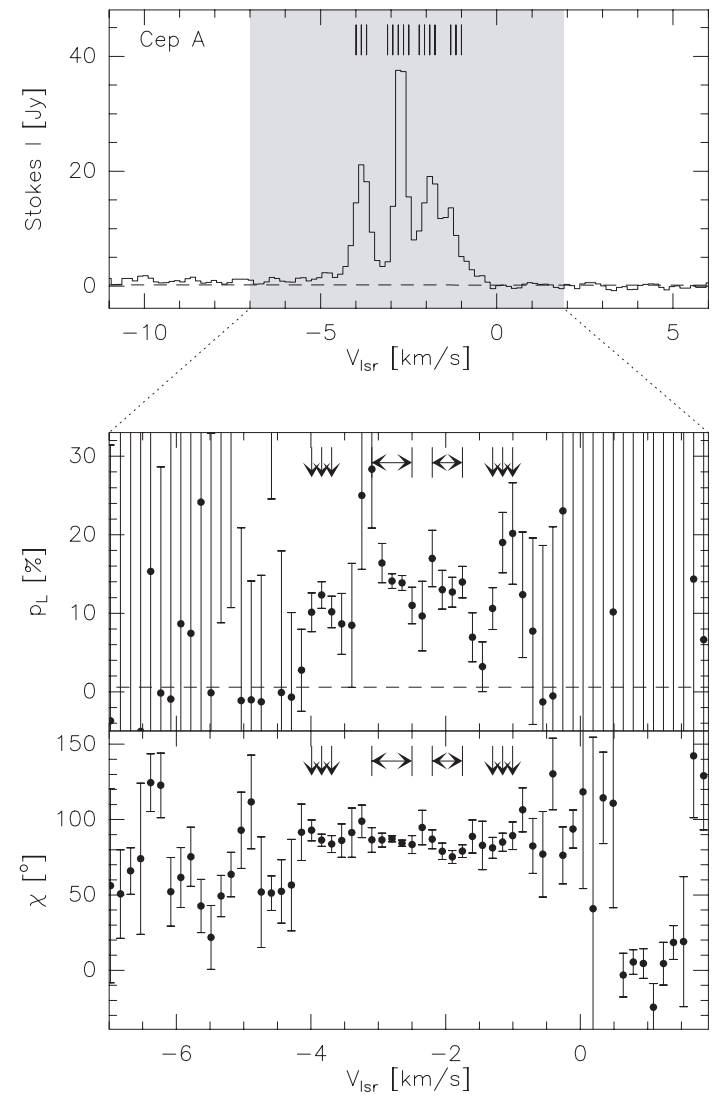

Fig. 6. Class II methanol masers at $157.0 \mathrm{GHz}$.

$\mathrm{CH}_{3} \mathrm{OH}$ maser rather emerges close to the thermal radio jet of Torrelles et al. (1996). Codella et al. (2003) found evidence of a well collimated outflow towards the south; the methanol maser could be located just at its onset.

\subsubsection{G $9.62+0.19$}

This maser has two components on top of a broad thermal and possibly quasi-maser contribution. As in the case of Cep A and NGC 7538-IRS1, both velocity components of this maser have the same polarization characteristics (weighted mean $p_{\mathrm{L}}=$ $33.0 \%$, at $\chi=-140 \% 6$ ). The masers' position and velocity coincide with that of a cluster ( $0 . .5$ in extent) of $6.7 \mathrm{GHz}$ maser emission (Norris et al. 1993). The associated UC HII region of this methanol maser is continuum source E (Garay et al. 1993), located near the edge of a compact, dense and warm molecular cloud core (Hofner et al. 1996). Hot thermal methanol emission of the $15_{3} \rightarrow 14_{4} A^{-}$line was imaged with the Plateau de Bure interferometer by Hofner et al. (2001). It coincides with the continuum source $\mathrm{E}$ and the maser position. Although the thermal methanol emission is point-like in the interferometer map, there may be an extended component missed by the interferometer. As a matter of fact, the whole line profile at $157 \mathrm{GHz}$ is polarized, which may be due to the sidelobe polarization of a component that is resolved in our beam.
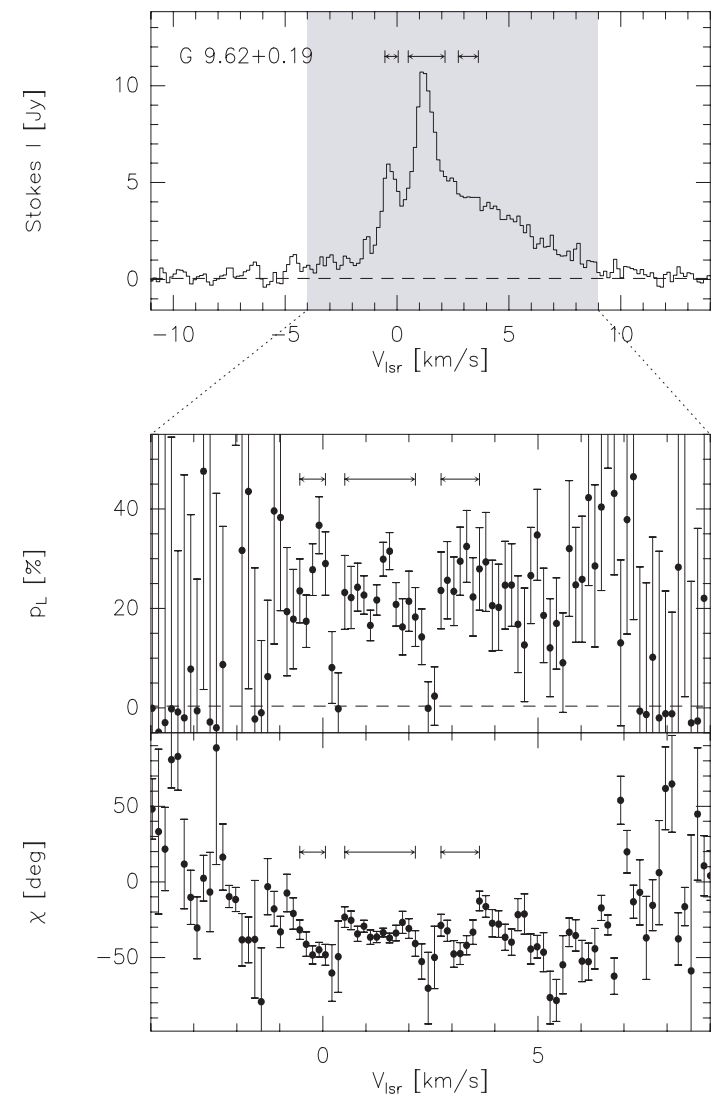

\section{Discussion}

Most models of maser polarization to date have been for saturated masers (Goldreich et al. 1973; Watson \& Wyld 2001). The polarization in such models is due to unequal populations in Zeeman-split substates of the maser levels and one finds that a high degree of maser saturation as well as the presence of a magnetic field is a necessary condition for linear polarization in this type of model. One can also have polarization in other situations, where the radiation field pumping the maser or the optical depth in FIR transitions connected to one of the maser levels is anisotropic. Western \& Watson (1983, 1984) investigated the role of anisotropic pumping in the polarization of maser lines, both for $J=1-0$ and $J=2-1$ transitions. Elitzur (1993) considered the exponential maser growth in the unsaturated phase, where the polarization is produced. Subsequently, it has been found (Elitzur 1996) that for nonparamagnetic molecules, where linewidths exceed the Zeeman splitting, the higher-order terms of the Zeeman splitting, although small, cannot be neglected, but have to be taken into account in order to rule out unphysical solutions. A summary of this work can be found in Elitzur (2002).

In the case of the masers observed by us, we feel that one should presently consider all options open and concentrate on obtaining better observational constraints on the physical conditions in the regions where methanol maser polarization is observed. Below we comment briefly both on what is 
known about the physical conditions in the clumps giving rise to methanol masers as well as on some of the current models.

\subsection{Physical conditions in the regions giving rise to methanol maser emission}

Studies of class I methanol masers to date have been hampered by poorly known collisional rates. However, a recent study by Pottage, Flower \& Davis $(2002,2004)$ of $\mathrm{He}-\mathrm{CH}_{3} \mathrm{OH}$ collisions has improved the situation, and it appears as if the estimates used in previous studies (e.g., Cragg et al. 1992; Johnston et al. 1992) were broadly correct. Calculations by Leurini et al. (2004) using the new rates confirm this.

On the basis of the earlier rate estimates, Johnston et al. found, using VLA observations of the $25 \mathrm{GHz}$ transitions towards Orion, that the $\mathrm{H}_{2}$ density in the maser region should be between $2 \times 10^{6}$ and $10^{8} \mathrm{~cm}^{-3}$ and the methanol column density in the range $2 \times 10^{16}$ to $2 \times 10^{17} \mathrm{~cm}^{-2}$. The temperature is expected to be of order $100 \mathrm{~K}$ and the inferred methanol abundance $\mathrm{CH}_{3} \mathrm{OH} / \mathrm{H}_{2} 10^{-7}$ to $10^{-6}$. These results are similar to those obtained for other $\mathrm{CH}_{3} \mathrm{OH}$ lines in other class I sources (e.g., Liechti \& Walmsley 1997, in the case of DR21) and one concludes that high density (above $10^{5} \mathrm{~cm}^{-3}$ ), high temperature (of order $100 \mathrm{~K}$ ), and high methanol abundance are characteristics of class I methanol emission. It is notable moreover that the estimated abundances are similar to those found by Bachiller et al. (1995) towards outflow regions, but much higher than those in cold dark clouds (Friberg et al. 1988). One interpretation of this is that the elevated methanol abundance is caused by sputtering of ice mantles of dust grains containing methanol in shocks associated with the outflows. If so, the alignment due to the shock likely has consequences for the magnetic field orientation and for the escape probability in optically thick $\mathrm{CH}_{3} \mathrm{OH}$ lines.

Class II methanol masers (in particular the 6.7 and 12.2 GHz transitions) have been studied by Sobolev et al. (1997a, 1997b) and by Cragg et al. (2002). The general conclusion is that the masers form in high density regions $\left(10^{5}\right.$ to $10^{8} \mathrm{~cm}^{-3}$ ) under conditions in which the gas temperature is lower than the dust temperature (both being of order $100 \mathrm{~K}$ ). There is evidence for association with $\mathrm{OH}$ maser regions having milligauss magnetic fields. There is evidence also that in $\mathrm{W} 3 \mathrm{OH}$, the maser regions are associated with absorption components in non-masing transitions of $\mathrm{OH}$ (Guilloteau et al. 1985) and methanol (Menten et al. 1986) whose temperatures are of the order $100-200 \mathrm{~K}$. In both cases $\left(\mathrm{CH}_{3} \mathrm{OH}\right.$ and $\left.\mathrm{OH}\right)$, one requires high abundances in the range $10^{-6}$ to $10^{-7}$ whose explanation (Hartquist et al. 1995) is thought to lie in the evaporation of $\mathrm{CH}_{3} \mathrm{OH}$ from the surface of grains heated by the passage of weak shocks. As a matter of fact, ISO observations (Gibb et al. 2000) have shown that $\mathrm{CH}_{3} \mathrm{OH}$ is an important constituent of interstellar ices associated with protostellar objects such as W 33 A. For low mass stars, Pontoppidan et al. (2003) detect abundant solid methanol, too.

\subsection{Models of polarization of saturated masers}

Goldreich et al. (1973) showed that the Zeeman splitting $\Omega_{\mathrm{B}}$ in polarized masers has to exceed $\sqrt{R \Gamma}$, where $R$ is the stimulated emission rate of the masing transition, and $\Gamma$ is the decay rate out of the maser levels. However, this result only holds for $J=1-0$ transitions. For higher angular momenta, Deguchi \& Watson (1990) showed that this condition has to be replaced by the stronger requirement $\Omega_{\mathrm{B}}>R$. It is difficult to get even a rough estimate of $R$ from observable quantities, since the spot size of $\mathrm{mm} \mathrm{CH}_{3} \mathrm{OH}$ masers is not well known. Lonsdale et al. (1998) deduce from mm VLBI measurements a lower limit of several milliarcseconds. In the following, we assume that $R \lesssim \Gamma$, as for unsaturated maser emission. We estimate for the gas density in $\mathrm{CH}_{3} \mathrm{OH}$ masers values of $10^{6}-10^{7} \mathrm{~cm}^{-3}$, where magnetic fields of around a $1 \mathrm{mG}$ are expected. The Zeeman splitting is thus about $0.3 \mathrm{~Hz}$. For the Landé factor of $\mathrm{CH}_{3} \mathrm{OH}$, a value of 0.078 has been used (Jen 1951), or equivalently $4.2 \times 10^{-5}$ if the Bohr magneton is used instead of the nuclear magneton. The loss rate $\Gamma$, on the other hand, is around $10^{-4}-10^{-3} \mathrm{~s}^{-1}$, for a collision rate of $10^{-10} \mathrm{~cm}^{-3} n_{\mathrm{H}_{2}}$, and the above gas density. The Zeeman splitting thus exceeds $\mathrm{R}$ by several orders of magnitude, except if the maser is strongly saturated (which is not the case here).

According to Goldreich et al. (1973), the polarization angle is either perpendicular to the projection of the magnetic field on the sky (hereafter $\boldsymbol{B}_{\text {sky }}$ ), if $\sin ^{2} \Theta>2 / 3$, where $\Theta$ is the angle between the magnetic field direction and the maser propagation direction, or parallel otherwise. However, this result only holds in the limiting case of extreme saturation $\Gamma \ll R$ and magnetic fields satisfying $R \ll \Omega_{\mathrm{B}}$.

Extensions of this model were studied by Nedoluha \& Watson (1990, hereafter NW90), including lower saturation and larger angular momenta. Since they are relevant for our results, we discuss them in the next section.

\subsection{Extensions of the idealizing maser polarization models}

NW90 (their Figs. 3a,b) showed that for all saturation parameters $R / \Gamma$ and for a maser propagation with $\sin ^{2} \Theta>2 / 3$ (yielding the largest linear polarization) the polarization angle remains (except for a small deflection) roughly perpendicular to $\boldsymbol{B}_{\text {sky }}$. For smaller $\Theta$, all orientations of the polarization angle, from parallel to $\boldsymbol{B}_{\text {sky }}$ for weak saturation to perpendicular to $\boldsymbol{B}_{\text {sky }}$ for strong saturation, are possible. For a $J=2-1$ transition, the polarization is lower than for a $J=1-0$ transition. We thus infer that the large polarizations ( $\gtrsim 10 \%)$ observed by us towards the class I masers would require $R \sim 100 \Gamma$, intermediate magnetic fields $\left(\Omega_{\mathrm{B}} / \Gamma \sim 100\right)$ and propagation preferentially perpendicularly to the magnetic field (cf. Figs. 3c,d of NW90). A stronger polarization can only be achieved by considering bidirectional propagation. According to NW90, for $J=2-1$ transitions, $R \sim \Gamma$, and propagation perpendicularly to the magnetic field, $\sim 10 \%$ linear polarization can be reached (for $\Omega_{\mathrm{B}} \geq 10 \Gamma$ ).

In unsaturated masers, the largest fractional polarizations observed by us ( $\left.p_{\mathrm{L}} \sim 30-40 \%\right)$ can be reached by means of 
anisotropic pumping (Western \& Watson 1984; see however Elitzur 1996, where maser polarization arises in the unsaturated growth phase). The radiation field that pumps $\mathrm{CH}_{3} \mathrm{OH}$ class II masers is naturally anisotropic when it comes e.g. from a nearby star, or when the escape probability for the pump radiation is anisotropic. As remarked above, the latter situation may occur e.g. in a layer compressed by a shock. Anisotropic pumping provides a substantial polarization: even for $J_{\text {up }}>1$, and $R \sim 10^{-3} \Gamma$ (unsaturated regime), and without magnetic fields, NW90 find, for axially symmetric pumping, a fractional linear polarization of $30-40 \%$, with a position angle along and a maser propagation perpendicular to the symmetry axis of the anisotropic pumping.

For class I masers, thought to be collisionally excited (Cragg et al. 1992), anisotropic pumping is possible if the collisions themselves are anisotropic. As suggested by NW90, such conditions may be encountered when the collision partners are charged particles undergoing ambipolar diffusion in a shock. However, it is uncertain whether the ionization fractions in maser regions are sufficient to account for anisotropic collisional pumping.

In any case, we can also expect polarization due to an anisotropic loss rate $\Gamma$, for both maser classes. As mentioned above, the anisotropic absorption of IR photons from the maser levels, populating torsionally excited states, will imply an unequal population of the magnetic substates of the maser levels.

\subsection{Application to the observed $\mathrm{mm} \mathrm{CH}_{3} \mathrm{OH}$ masers}

It is very likely that most of our $\mathrm{CH}_{3} \mathrm{OH}$ masers are unsaturated, given their weakness with respect to the lower frequency transitions of the same class. Unfortunately, determining the degree of maser saturation is notoriously difficult. In the following, we assume that the observed $\mathrm{mm} \mathrm{CH}_{3} \mathrm{OH}$ masers are unsaturated. As discussed above, anisotropic pumping or anisotropic radiative losses are then a necessary condition to achieve the observed linear polarization.

Although a quantitative analysis of $\mathrm{CH}_{3} \mathrm{OH}$ maser polarization is still lacking, we can discuss our results in view of the models presented in the previous section. Figure 1 shows the distribution of detections of fractional polarization among the sources listed in Tables 2 and 3. The highest linear polarizations (35-40\%) are reached in both classes. Although our sample is still too small to derive reliable statistics from it, we find that a large fractional linear polarization is regularly achieved in both classes of $\mathrm{mm} \mathrm{CH}_{3} \mathrm{OH}$ masers. Whether anisotropic pumping and anisotropic radiative losses are needed or not to achieve such polarizations is a matter of current debate.

\subsection{Discussion of individual sources}

\subsubsection{OMC-2}

The direction of the linear polarization of the $133 \mathrm{GHz}$ class I maser in OMC 2 is close to the outflow axis (Fig. 7). The outflow direction is well defined both by $\mathrm{CO}$ observations (Williams et al. 2003) and by the alignment of the $44 \mathrm{GHz}$ maser spots (Kogan \& Slysh 1998). Since a shock at the

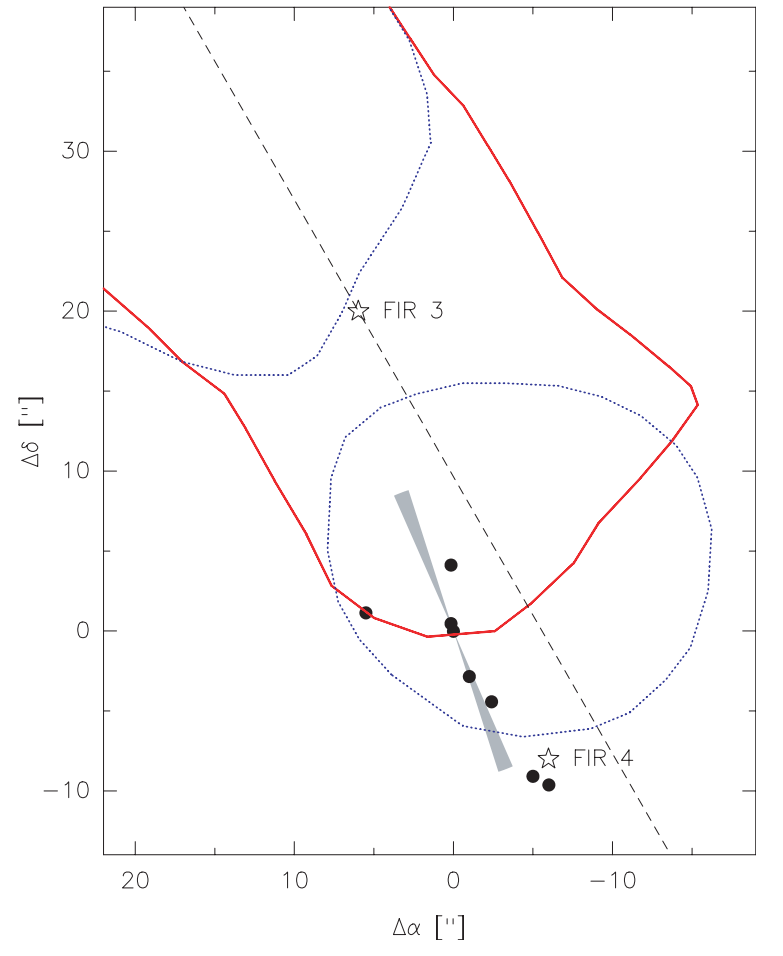

Fig. 7. Schematic representation of methanol masers in OMC-2. Black dots: $44 \mathrm{GHz}$ masers of Kogan \& Slysh (1998). Contours: extent of the overlapping outflow system (in CO(1-0), from Williams et al. 2003). The dashed straight line is the outflow axis. Dotted contours: blue shifted lobes. Solid contour: red shifted lobe. The polarization angle of our $133 \mathrm{GHz}$ measurement and its error are indicated by the shadowed line (whose length is the beamwidth, FWHM). The positions of the FIR sources are from Chini et al. (1997).

"working surface" of the outflow will be too fast and destroy molecules, an oblique shock, propagating away from the outflow axis, explains both the required enhanced gas-phase $\mathrm{CH}_{3} \mathrm{OH}$ abundance, and the asymmetry for an anisotropic loss rate (i.e. escape probability for IR photons). In this scenario, the magnetic field tends to line up roughly along the shock front, and only a maser propagation parallel to the shock plane will provide a strong maser gain. The magnetic field and the maser propagation can then be almost perpendicular, because this outflow is not viewed pole-on. Such a model provides an explanation for both the observed fractional linear polarization ( $p_{\mathrm{L}}=7 \%$ despite $R \lesssim \Gamma$ ) and its orientation parallel to $\boldsymbol{B}_{\text {sky }}$ and hence to the outflow axis (cf. NW90, their Fig. 11, case (2)).

\subsubsection{DR21 -W}

The case of DR21 -W is less clear. Slysh et al. (1997) observed that the $84 \mathrm{GHz}$ and $133 \mathrm{GHz}$ lines have similar spectral features. All the more it is astonishing that in DR 21-W, both masers, similar in strength, line shape and fractional linear polarization have polarization angles that differ by $40^{\circ}$. The reason may simply be the variability of unsaturated maser emission (the $84 \mathrm{GHz}$ maser was observed in February 2002, the one at $133 \mathrm{GHz}$ in May 2002). If the polarization characteristics are constant in time, and if the spatial separation of the masers at $84 \mathrm{GHz}$ and $133 \mathrm{GHz}$ is similar to that of the two $44 \mathrm{GHz}$ 
components of Kogan \& Slysh, this could be a hint at an inhomogeneous magnetic field and/or an anisotropic pump rate $P$ or loss rate $\Gamma$ with different symmetry axes for both masers (for a distance of $3 \mathrm{kpc}, 2$ '" 8 correspond to 8000 AU, cf. Plambeck \& Menten 1990, further references therein). However, the spectral profiles of the polarization angle of either source is flat, i.e. the magnetic field and/or $P$ and $\Gamma$ are homogeneous at least along each maser's axis.

\subsubsection{NGC 7538}

In NGC 7538-Met1, the linear polarization is roughly perpendicular to a suggestive bow-shock structure as traced by shockexcited $\mathrm{H}_{2}$ emission (Davis et al. 1998). This is schematically shown in Fig. 8. As for OMC-2, the polarization angle profile is rather flat, and a similar scenario may apply. The polarization angles of the $133 \mathrm{GHz}$ class I maser towards NGC 7538-Met1 and of the $107 \mathrm{GHz}$ class II maser towards NGC 7538-IRS1 are very close to those of the $\lambda 870 \mu \mathrm{m}$ continuum emission from these positions. Since the magnetic field direction in the dust emitting region and the maser region is likely to be the same, this may be taken as evidence for magnetic alignment of dust grains. However, in the unsaturated regime, it cannot be ruled out that the linear polarization is rather due to the amplification of polarized background radiation from dust. Then the fractional linear polarization of the maser radiation and its polarization angle are the same as for the continuum seed radiation (see, e.g., Goldreich et al. 1973, Eq. (64); or Wallin \& Watson 1997, Eqs. (17)-(19)), whatever the explanation of the grain alignment may be. This is only valid if the magnetic substates are equally populated, i.e. the maser has to be weak, so that its own radiation does not yet affect the absorption coefficients. In order to fulfill this condition, we have to replace the inequality $R \ll \Gamma$ by the more stringent $R / \Gamma \ll \Omega_{\mathrm{B}} / \Delta \omega_{\mathrm{D}}$, where $\Delta \omega_{\mathrm{D}}$ is the Doppler linewidth. For a $10 \mathrm{mG}$ field, and a mean maser linewidth of $0.5 \mathrm{~km} \mathrm{~s}^{-1}$ (FWHM), we have $\Omega_{\mathrm{B}} / \Delta \omega_{\mathrm{D}} \sim 10^{-5}$, since the $\mathrm{CH}_{3} \mathrm{OH}$ molecule is non-paramagnetic. In turn, $R \ll$ $10^{-5} \Gamma$ is only valid for rather weak masers, otherwise the polarization due to the seed radiation will be destroyed by the (still unsaturated) maser itself. An observable quantity which allows to distinguish between both regimes is the line-to-continuum flux ratio. Since this ratio was only measurable with limited accuracy and spatial resolution towards two sources (see Sect. 3), the data presented here do not yet allow for a meaningful conclusion. Interestingly, Pestalozzi et al. (2004) successfully model the 12.2 and $6.7 \mathrm{GHz}$ class II methanol maser towards NGC 7538-IRS1 by high amplification of background radio continuum emission.

\subsection{Spectral profiles of the polarization angle}

Most of the polarized class I masers have flat polarization angle profiles. Only two sources (S 231 and W 51-Met 2) have an almost constant slope (Fig. 9), of $-72^{\circ} 8 / \mathrm{km} \mathrm{s}^{-1}$ and $97.6 / \mathrm{km} \mathrm{s}^{-1}$, respectively. As shown by NW90, a slope in the polarization angle profile is produced by a gradient in the magnetic field direction, for a constant loss rate $\Gamma$ and pump rate $P$.

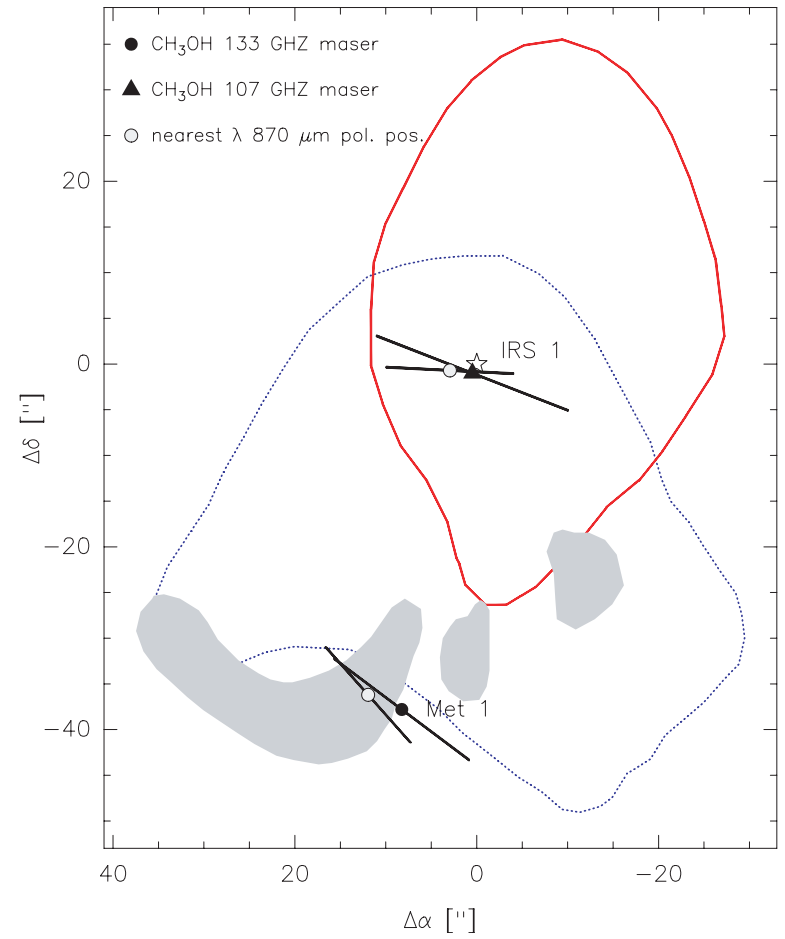

Fig. 8. Schematic representation of the $\mathrm{mm}$ methanol masers in NGC 7538, centered at the position of IRS 1. Contours: extent of the outflow system (in CO(1-0), from Kameya et al. 1989). Dotted contour: blue shifted lobe. Solid contour: red shifted lobe. The polarization angle of our $107 \mathrm{GHz}$ and $133 \mathrm{GHz} \mathrm{CH} \mathrm{CH}_{3} \mathrm{OH}$ maser measurements (filled circle resp. filled triangle) as well as that of the nearest $\lambda 870 \mu \mathrm{m}$ continuum polarization measurements (open circles, Momose et al. 2001) are indicated by the straight lines (whose lengths indicate the beamwidth, FWHM). The grey-shaded area indicates the $\mathrm{H}_{2}$ emission suggesting a bow shock (Davis et al. 1998).

Consequently, the spectral profile of the linear polarization has a maximum at the velocity where the angle between the magnetic field and the maser propagation direction is largest (clearly visible in S 231 at $95 \mathrm{GHz}$, Fig. 3).

Keplerian disks with an azimuthal, frozen-in magnetic field component following the velocity vectors (as modelled by Barvainis 1984, for the $\mathrm{SiO}$ maser in Orion IRc 2) are an attractive model providing an unconstrained explanation for both a gradient of the magnetic field direction, and for an anisotropic pump or loss rate. For the case of the $6.7 \mathrm{GHz}$ class II maser in S 231, Minier et al. (2000) propose a masing disk as explanation of the kinematics of the maser spots. Pestalozzi et al. (2004) explain the main component of the 12.2 and $6.7 \mathrm{GHz}$ $\mathrm{CH}_{3} \mathrm{OH}$ maser emission towards NGC 7538-IRS1 by a differentially rotating disk. On the other hand, De Buizer (2003) casts doubt on the disk interpretation, finding that the shockexcited $\mathrm{H}_{2}$ emission towards young stellar objects associated with linearly distributed methanol masers is mostly aligned both with the associated outflows and with the methanol maser spots (which should be aligned perpendicularly to the outflow in the disk scenario). After all, a slope in the polarization angle profile can also simply be due to a changing direction of the symmetry axis for an anisotropic pump or loss rate, without any need for a particular magnetic field structure. Only more 

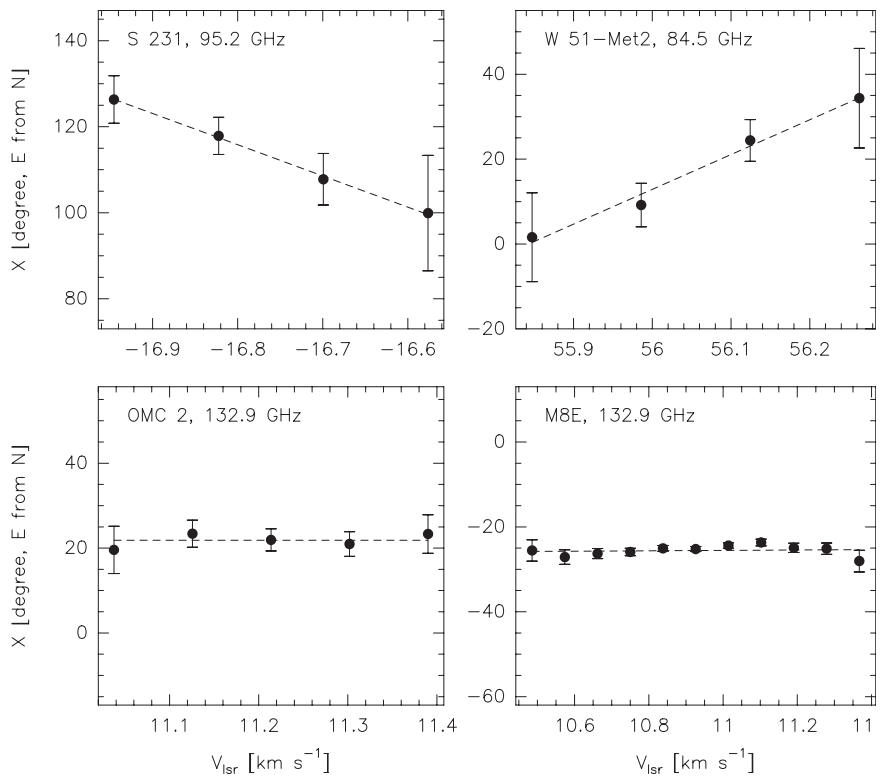

Fig. 9. Polarization angle profiles of class $\mathrm{I} \mathrm{CH}_{3} \mathrm{OH}$ masers. The ordinates are all at the same scale, to better compare the end-to-end difference of polarization angles. The flat profiles (bottom row) are clearly distinguished from those with a slope (top row).

accurate models and higher spatial resolution observations will allow to distinguish between those scenarios.

\subsection{Circular polarization}

The tentative detection of circular polarization in two class I methanol masers (Fig. 10) is interesting and the mechanism producing the effect is not clear. We note that the Zeeman components of the $\mathrm{CH}_{3} \mathrm{OH}$ maser are unresolved: as estimated in Sect. 4.5.3, $\Omega_{\mathrm{B}} / \Delta \omega_{\mathrm{D}} \sim 10^{-5}$. Due to the unsaturated maser emission, the circular polarization observed in the $133 \mathrm{GHz}$ masers of M8E and DR 21-W can only be due to non-Zeeman effects, a conclusion also supported by the absence of the classical S-shaped signature of Stokes $V$. As estimated above, we have $\Omega_{\mathrm{B}} \gg R$ and $\Gamma$, and the symmetry axis for the molecular quantum substates ("quantization axis") in the masing medium is aligned with the magnetic field. Circular polarization in this regime was investigated by Wiebe \& Watson (1998), and results from a misalignment of the local quantization axis and the direction of linear polarization. Such a misalignment can be achieved by changes of $\boldsymbol{B}_{\text {sky }}$ along the maser propagation path. Towards class II masers, we did not detect circular polarization. Our most stringent upper limit is $p_{\mathrm{C}}<2.5 \%$ (for NGC 7538-IRS1, 107 GHz maser). Likewise, Koo et al. (1988) get a negative result from their survey of the $12 \mathrm{GHz}$ line.

\subsection{The velocity components of class II masers}

Another remarkable result is that the different velocity components of a given class II maser, although different in flux density, display a similar fractional polarization and polarization angle. This suggests either masers that are not too far from each other (e.g. $500 \mathrm{AU}$ in the case of the components A and B
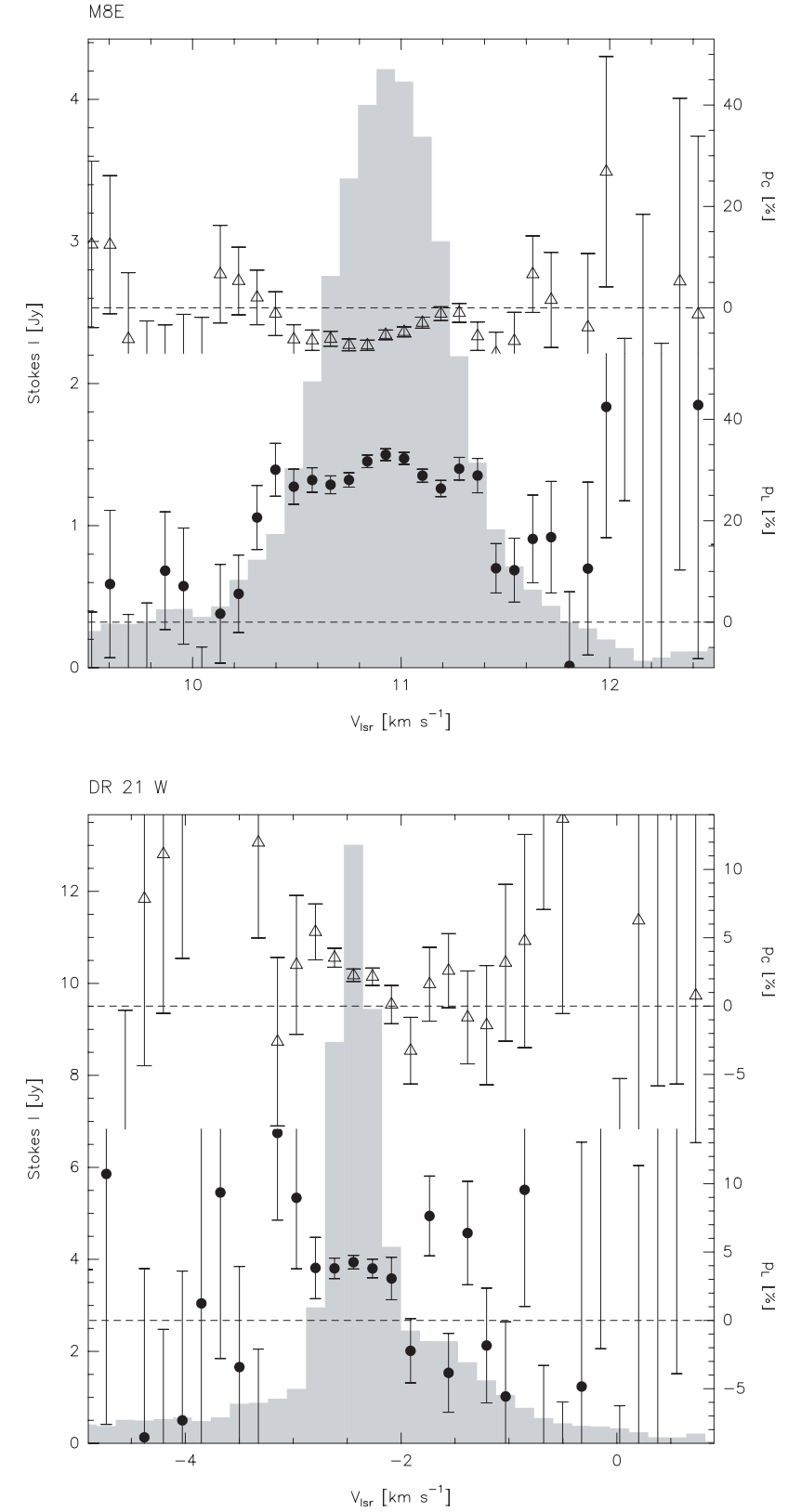

Fig. 10. Detections of circular polarization towards M8E (upper) and DR 21-W (lower), both in the $133 \mathrm{GHz} \mathrm{CH} \mathrm{CH}_{3} \mathrm{OH}$ maser. The circular polarization $p_{\mathrm{C}}$ is indicated by open triangles, and the linear polarization $p_{\mathrm{L}}$ by filled dots (both on the same scale). The dashed lines indicate the level of zero polarization. For comparison, the Stokes $I$ spectra are shown as grey-shaded histograms. The axes of M8E and DR 21-W are not on the same scale.

of Cep A East, see Fig. 11), or that the velocity of a single maser is redistributed along its propagation direction, splitting the maser line into different spectral components (e.g. in Fig. 11 the components labelled A in Cep A East, that are spatially and spectrally unresolved by Mehringer et al. 1997). In both cases, we can expect a rather homogeneous magnetic field structure and/or distribution of the anisotropy of the pumping radiation field. As for velocity redistribution, Nedoluha \& Watson (1988) show that such a breakup into multiple, narrow lines is even possible in presence of a smooth velocity gradient of the 


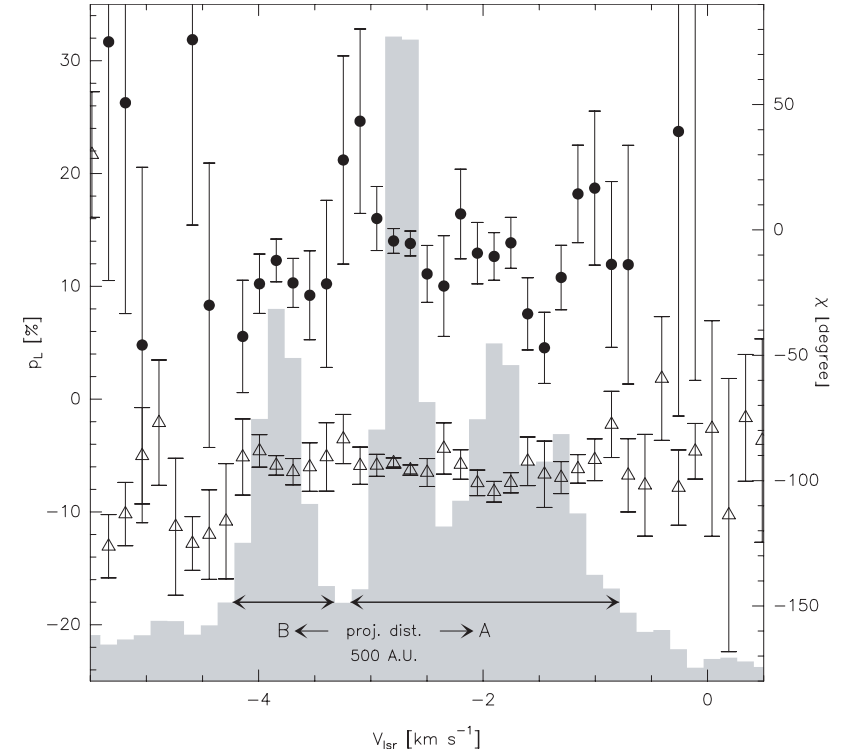

Fig. 11. Overlay of the linear polarization (filled circles, left ordinate) and the polarization angle (unfilled triangles, right ordinate) with the spectrum (grey-shaded) of the $157 \mathrm{GHz} \mathrm{CH} \mathrm{CH}_{3} \mathrm{OH}$ maser towards Cep A. The projected distance of the components A and B is indicated (derived by Mehringer et al. 1997).

order of a few $\mathrm{km} \mathrm{s}^{-1}$ along the maser. Despite these changes in velocity, the magnetic field and - perhaps more importantly the direction of the pumping anisotropic radiation field remain constant. A constant anisotropy parameter is expected if the maser length is small compared to the distance from the source of pump radiation.

\section{Conclusions}

1. We find linear polarization in 10 out of 14 class I sources, and in 3 out of 7 class II sources. The highest polarization $(39.5 \%)$ is found in the class I maser L 379, the most significant one in $\mathrm{M} 8 \mathrm{E}\left(p_{\mathrm{L}} / \sigma_{\mathrm{rms}}>25\right.$, both at $133 \mathrm{GHz}$ ). Both class I and class II $\mathrm{mm} \mathrm{CH} \mathrm{CH}_{3} \mathrm{OH}$ masers reach substantial polarizations well above $10 \%$. The $\mathrm{mm} \mathrm{CH}_{3} \mathrm{OH}$ masers, given their weakness relative to those at lower frequencies, are unsaturated. The polarization observed possibly needs, especially for the larger angular momenta, anisotropic pumping and an anisotropic escape probability leading to an anisotropic loss rate. The maximal polarization of class I masers is close to that observed for $\mathrm{H}_{2} \mathrm{O}$ masers. None of our three polarized class II sources reaches a polarization as high as that of $\mathrm{SiO}$ masers.

2. For class I masers, the polarization angle profiles across the maser lines are mostly flat; two sources (W51-Met 2 and $S$ 231) show evidence of a linear slope, suggesting that the direction of the magnetic field and of the symmetry axis for anisotropic loss or pumping changes along the propagation axis of the maser. The more common flat polarization angle profiles could be attributed to masers originating from shocks that are seen edge-on, providing the largest possible gain lengths and a highly anisotropic escape probability.
3. The polarization angles of the class I and II masers in NGC 7538 show a remarkable agreement with the results from submillimeter continuum polarization, the latter being generated by magnetically aligned grains. We suggest that the class I maser is associated with a bow shock in the outflow. The magnetic field in this maser is likely enhanced by compression; due to the shock, the loss rate is anisotropic, yielding a $133 \mathrm{GHz}$ maser with a relatively strong polarization of 24\%. The $107 \mathrm{GHz}$ class II maser towards IRS1 is weakly polarized. For both masers, the polarization angles are close to those of the submm continuum towards the respective positions of the masers. This provides evidence for magnetic alignment of dust grains, the magnetic fields in the dust emitting and masing volumes having the same direction. Another explanation is the amplification of the polarized continuum emission, which provides the seed photons for this unsaturated maser. In several of the polarized class I and class II $\mathrm{mm} \mathrm{CH}_{3} \mathrm{OH}$ masers shown here, the line-to-continuum flux ratio is sufficiently small to make such a scenario plausible.

4. The polarization direction of the prominent class I maser in OMC-2 is close to the outflow axis. This observation can be interpreted as a maser originating in a lateral C-type shock compressing the gas in a plane roughly parallel to the outflow axis, making the loss rate anisotropic. The linear polarization - relatively strong for an unsaturated maser - indicates a maser propagation perpendicularly to the magnetic field. The flat spectral profile of the polarization angle lends additional support to this shock scenario.

5. All class II masers where we detect polarization have several (at least two) velocity components with the same polarization characteristics. This is indicative of velocity redistribution along the propagation direction of a single maser with a velocity gradient, and/or of nearby, but unresolved individual masers with a constant magnetic field direction and a constant direction for anisotropic pumping or radiative losses.

6. Significant circular polarization of $-7.1 \%$ and $3.5 \%$ was tentatively detected towards two class I masers at $133 \mathrm{GHz}$ (M8E respectively DR 21-W). We attribute this result to a non-Zeeman origin, due to the weakness of the Zeeman splitting with respect to the linewidth of the $\mathrm{CH}_{3} \mathrm{OH}$ emission (the molecule is non-paramagnetic), and because the maser emission is unsaturated.

Acknowledgements. We thank our IRAM colleagues M. Torres who built the principal component of the IF polarimeter, and D. Morris who participated in the development and commissioning of the polarimeter. We are also grateful to our Spanish IRAM colleagues S. Navarro, who helped us to install the polarimeter, G. Paubert, who configured the backends for it, and A. Sievers and the Pico Veleta staff who provided assistance with the observations. We acknowledge the comments of an anonymous referee.

\section{References}

Allen, D. A. 1972, ApJ, 172, L55

Anderson, T., de Lucia, F., \& Herbst, E. 1990, ApJS, 72, 797

Bachiller, R., Liechti, S., Walmsley, C. M., \& Colomer, F. 1995, A\&A, 295, L51 
Barvainis, R. 1984, ApJ, 279, 358

Batrla, W., \& Menten, K. M. 1988, ApJ, 329, L117

Caswell, J. L., Gardner, F. F., Norris, R. P., et al. 1993, MNRAS, 260, 425

Chini, R., Reipurth, B., Ward-Thompson, D., et al. 1997, ApJ, 474, L135

Chrysostomou, A., Aitken, D. K., Jenness, T., et al. 2002, A\&A, 385, 1014

Codella, C., Bachiller, R., Benedettini, M., \& Caselli, P. 2003, MNRAS, 341, 707

Cragg, D. M., Johns, K. P., Godfrey, P. D., \& Brown, R. D. 1992, MNRAS, 259, 203

Cragg, D. M., Sobolev, A. M., \& Godfrey, P. D. 2002, MNRAS, 331, 521

Davis, C. J., \& Smith, M. D. 1996, A\&A, 310, 961

Davis, C. J., Moriarty-Schieven, G., Eislöffel, J., Hoare, M. G., \& Ray, T. P. 1998, AJ, 115, 1118

De Buizer, J. M. 2003, MNRAS, 341, 277

Deguchi, S., \& Watson, W. D. 1990, ApJ, 354, 649

Elitzur, M. 1993, ApJ, 416, 256

Elitzur, M. 1996, ApJ, 457, 415

Elitzur, M. 2002, Astrophysical Spectropolarimetry, ed. J. Trujillo-Bueno et al. (Cambridge Univserity Press), 225

Friberg, P., Hjalmarson, A., Madden, S. C., \& Irvine, W. M. 1988, A\&A, 195, 281

Garay, G., Rodriguez, L. F., Moran, J. M., \& Churchwell, E. 1993, ApJ, 418, 368

Garden, R. P., Geballe, T. R., Gatley, I., \& Nadeau, D. 1991, ApJ, 366, 474

Gibb, E. L., Whittet, D. C. B., Schutte, W. A., et al. 2000, ApJ, 536, 347

Goldreich, P., Keeley, D. A., \& Kwan, J. Y. 1973, ApJ, 179, 111

Goldreich, P., \& Kylafis, N. D. 1982, ApJ, 253, 606

Guilloteau, S., Baudry, A., \& Walmsley, C. M. 1985, A\&A, 153, 179

Hartquist, T. W., Menten, K. M., Lepp, S., \& Dalgarno, A. 1995, MNRAS, 272, 184

Hodapp, K. W., \& Davis, C. J. 2002, ApJ, 575, 291

Hofner, P., Kurtz, S., Churchwell, E., Walmsley, C. M., \& Cesaroni, R. 1996, ApJ, 460, 359

Hofner, P., Wiesemeyer, H., \& Henning, T. 2001, ApJ, 549, 425

Jen, C. K. 1951, Phys. Rev., 81, 197

Johnston, K. J., Gaume, R., Stolovy, S., et al. 1992, ApJ, 385, 232

Kameya, O., Hasegawa, T. I., Hirano, N., Takakubo, K., \& Seki, M. 1989, ApJ, 339, 222

Kelly, M. L., \& MacDonald, G. H. 1996, MNRAS, 282, 401

Kogan, L., \& Slysh, V. 1998, ApJ, 497, 800

Koo, B., Williams, D. R. D., Heiles, C., \& Backer, D. C. 1988, ApJ, 326, 931

Leach, R. W., Clemens, D. P., Kane, B. D., \& Barvainis, R. 1991, ApJ, 370,257

Leurini, S., Schilke, P., Menten, K. M., et al. 2004, A\&A, 422, 573

Liechti, S., \& Walmsley, C. M. 1997, A\&A, 321, 625

Lonsdale, C. J., et al. 1998, BAAS, 30, 1355
Mehringer, D. M., Zhou, S., \& Dickel, H. R. 1997, ApJ, 475, L57

Menten, K. M., Walmsley, C. M., Henkel, C., et al. 1986, A\&A, 169, 271

Menten, K. M. 1991, ApJ, 380, L75

Minchin, N. R., \& Murray, A. G. 1994, A\&A, 286, 579

Minier, V., Booth, R. S., \& Conway, J. E. 2000, A\&A, 362, 1093

Mitchell, G. F., Hasegawa, T. I., \& Schella, J. 1992, ApJ, 386, 604

Momose, M., Tamura, M., Kameya, O., et al. 2001, ApJ, 555, 855

Nedoluha, G. E., \& Watson, W. D. 1988, ApJ, 335, L19

Nedoluha, G. E., \& Watson, W. D. (NW90) 1990, ApJ, 354, 660

Norris, R. P., Whiteoak, J. B., Caswell, J. L., Wieringa, M. H., \& Gough, R. G. 1993, ApJ, 412, 222

Pestalozzi, M. R., Elitzur, M., Conway, J. E., \& Booth, R. S. 2004, ApJ, submitted

Plambeck, R. L., \& Menten, K. M. 1990, ApJ, 364, 555

Pontoppidan, K. M., Dartois, E., van Dishoeck, E. F., Thi, W.-F., \& d'Hendecourt, L. 2003, A\&A, 404, L17

Pottage, J. T., Flower, D. R., \& Davis, S. L. 2002, J. Phys. B Atom. Mol. Phys., 35, 2541

Pottage, J. T., Flower, D. R., \& Davis, S. L. 2004, J. Phys. B Atom. Mol. Phys., 37, 165

Schreyer, K., Helmich, F. P., van Dishoeck, E. F., \& Henning, T. 1997, A\&A, 326, 347

Scoville, N. Z., Sargent, A. I., Sanders, D. B., et al. 1986, ApJ, 303, 416

Sievers, A. W., Mezger, P. G., Kreysa, E., et al. 1991, A\&A, 251, 231

Slysh, V. I., Kalenski, S. V., \& Val'tts, I. E. 1995, ApJ, 442, 668

Slysh, V. I., Kalenskii, S. V., Val'tts, I. E., \& Golubev, V. V. 1997, ApJ, 478, L37

Sobolev, A. M., \& Deguchi, S. 1994, A\&A, 291, 569

Sobolev, A. M., Cragg, D. M., \& Godfrey, P. D. 1997, MNRAS, 288, L39

Sobolev, A. M., Cragg, D. M., \& Godfrey, P. D. 1997, A\&A, 324, 211

Thum, C., Wiesemeyer, H., Morris, D., Navarro, S., \& Torres, M. 2003, Proc. SPIE, 4843, 272

Torrelles, J. M., Gomez, J. F., Rodriguez, L. F., et al. 1996, ApJ, 457, L107

Torrelles, J. M., Patel, N. A., Gomez, J. E., et al. 2001, Nature, 411, 277

Val'tts, I. E., Dzyura, A. M., Kalenskii, S. V., et al. 1995, Astron. Rep., 39,18

Val'tts, I. E., Dzura, A. M., Kalenskii, S. V., et al. 1995, A\&A, 294, 825

Val'tts, I. E. 1999a, Astron. Rep., 43, 149

Val'tts, I. E. 1999b, Astron. Rep., 43, 157

Wallin, B. K., \& Watson, W. D. 1997, ApJ, 481, 832

Wardle, J. F. C., \& Kronberg, P. P. 1974, ApJ, 194, 249

Watson, W. D., \& Wyld, H. W. 2001, ApJ, 558, L55

Western, L. R., \& Watson, W. D. 1983, ApJ, 275, 195

Western, L. R., \& Watson, W. D. 1984, ApJ, 285, 158

Wiebe, D. S., \& Watson, W. D. 1998, ApJ, 503, L71

Williams, J. P., Plambeck, R. L., \& Heyer, M. H. 2003, ApJ, 591, 1025

Wilson, T. L., \& Mauersberger, R. 1990, A\&A, 239, 305 